\title{
Solar evolution and extrema: current state of understanding of long-term solar variability and its planetary impacts
}

\author{
Dibyendu Nandy ${ }^{1,2^{*}}$ D, Petrus C. H. Martens ${ }^{3}$, Vladimir Obridko ${ }^{4}$, Soumyaranjan Dash ${ }^{1}$ and Katya Georgieva ${ }^{5}$
}

\begin{abstract}
The activity of stars such as the Sun varies over timescales ranging from the very short to the very long — stellar and planetary evolutionary timescales. Experience from our solar system indicates that short-term, transient events such as stellar flares and coronal mass ejections create hazardous space environmental conditions that impact Earthorbiting satellites and planetary atmospheres. Extreme events such as stellar superflares may play a role in atmospheric mass loss and create conditions unsuitable for life. Slower, long-term evolutions of the activity of Sunlike stars over millennia to billions of years result in variations in stellar wind properties, radiation flux, cosmic ray flux, and frequency of magnetic storms. This coupled evolution of star-planet systems eventually determines planetary and exoplanetary habitability. The Solar Evolution and Extrema (SEE) initiative of the Variability of the Sun and Its Terrestrial Impact (VarSITI) program of the Scientific Committee on Solar-Terrestrial Physics (SCOSTEP) aimed to facilitate and build capacity in this interdisciplinary subject of broad interest in astronomy and astrophysics. In this review, we highlight progress in the major themes that were the focus of this interdisciplinary program, namely, reconstructing and understanding past solar activity including grand minima and maxima, facilitating physical dynamo-model-based predictions of future solar activity, understanding the evolution of solar activity over Earth's history including the faint young Sun paradox, and exploring solar-stellar connections with the goal of illuminating the extreme range of activity that our parent star-the Sun-may have displayed in the past, or may be capable of unleashing in the future.
\end{abstract}

Keywords: Solar magnetic fields, Stellar evolution, Stellar flares, Stellar wind, Space weather, Space climate, Faint Young Sun paradox, Star planet interactions, Planetary evolution, Habitability

\section{Introduction}

The Sun is a typical middle-aged G-type star whose current age is 4.6 billion years. It is an active star, with its magnetic activity driving short-to-long-term fluctuations in its radiative, electromagnetic, and particulate output. Occasionally, magnetic structures in the Sun generate magnetic storms-flares and coronal mass ejections (CMEs) - that are accompanied by intense

\footnotetext{
* Correspondence: dnandi@iiserkol.ac.in

${ }^{1}$ Center of Excellence in Space Sciences India, Indian Institute of Science

Education and Research Kolkata, Mohanpur, West Bengal 741246, India

${ }^{2}$ Department of Physical Sciences, Indian Institute of Science Education and

Research Kolkata, Mohanpur, West Bengal 741246, India

Full list of author information is available at the end of the article
}

radiation output, solar energetic particles (SEPs) and magnetized plasma ejecta that perturb planetary space environments (Schwenn 2006; Pulkkinen 2007). Studies of short-term solar variability and their influence on space-reliant technologies and planetary atmospheres and magnetospheres, including efforts to understand and predict solar activity are collectively termed as the science of space weather (Schrijver et al. 2015). Slower, long-term fluctuations in solar output over decades to millennia are driven by long-term variations in the solar cycle which is powered by the dynamo mechanism that works in the solar interior (De Jager 2005). These variations create space climate-changing radiative and 
particulate environment in the heliosphere that can impact planetary atmospheres and climate ranging from decadal to longer timescales (Mursula et al. 2007; Nandy and Martens 2007; Versteegh 2005). On top of this variability manifest over few generations of humans, longer term stellar evolutionary processes drive changes in the solar convection zone (SCZ) and differential rotation that alter the nature of the solar dynamo, and sustain evolutionary changes in solar wind, solar radiation, and solar storm frequency which together determine space environmental forcing of planetary atmospheres over billions of years-i.e., the evolutionary history of planets (Nandy and Martens 2007).

While ground-based observations spanning four centuries have given us a fairly good idea of decadal to century-scale fluctuations in the solar magnetic cycle, and more recent space-based observations have uncovered subtle variations in solar radiation, constrained energetic processes such as flares and CMEs, and measured properties of the solar wind, one has to take recourse to observations of other Sun-like stars and exoplanetary systems to appreciate the full range of plausible activity levels across the history of a Sun-like star and its consequence of planets (Nandy and Martens 2007; Brun et al. 2015). The rapidly emerging field of Star-Planet Interactions opens up this window to explorations of the coupled evolution of star-planet systems such as the Sun-Earth system or exoplanets around active stars (Deeg and Belmonte 2018; Das et al. 2019).

In this context, the Scientific Committee on SolarTerrestrial Physics (SCOSTEP) supported the Solar Evolution and Extrema (SEE) program to address the interdisciplinary nature of the science that seeks to uncover and explore solar activity across a wide range of time scales so that one can fathom the impact of this activity on planet Earth and beyond. The SEE program sought to facilitate community research to broadly seek answers to the following set of problems: Are we at the verge of a new grand minimum? If not, what is the expectation for cycle 25? For the next few decades, what can we expect in terms of extreme solar flares and storms, and also absence of activity? What is the largest solar eruption/flare possible? What is the expectation for periods with low activity? Does our current best understanding of the evolution of solar irradiance and mass loss resolve the "Faint Young Paradox"? How does geospace response to extreme solar events such as superflare or energetic CMEs. The SEE program spawned various collaborative efforts, supported long-term data archives through the SCOSTEP program, and hosted several meetings over the course of 4 years in order to build capacity in this domain of research. Individual results from the SEE program are reported elsewhere (Obridko and Georgieva 2018). Here, we review the general state of the field that the SEE program targeted and summarize the current state of our understanding of solar activity evolution and extrema over timescales relevant for atmospheric forcing and planetary evolution. For purposes of readability and with the motivation to provide a seamless context, here we do not attempt to explicitly segregate research results obtained during and previous to the SEE era; rather, our focus is on providing the relevant context and current status that would inform and motivate future research in this domain.

\section{The solar activity cycle: past, present, and future}

The Sun is a middle-aged G-type star whose current age is 4.6 billion years. It is a moderately active star, with its magnetic activity driving short-to-long-term fluctuations in its activity output. The emergence of sunspots on the solar surface varies in a periodic fashion. This is referred to as the solar cycle, which originates in a magnetohydrodynamic (MHD) dynamo mechanism that is sustained by plasma motions in the Sun's interior. Much of the constraints on this dynamo mechanism come from observations of sunspots (Fig. 1)-dark, magnetized regions on the solar surface-whose magnetic field strength can reach a few thousand gauss $(\mathrm{G})$. Long-term observations over four centuries, since the invention of the telescope in the early 1600s, show that the number of sunspots observed on the Sun increases and decreases in a cyclic fashion (Schwabe 1844; Hathaway 2015), thereby generating the solar cycle (Fig. 2). The sunspots observed on the surface show a systematic tilt which increases with latitude. This is also known as Joy's law (Hale et al. 1919). In reality, the variation is quasiperiodic, with cycle periods ranging between 9 and 14 years with a mean period of 11 years and strong fluctuations in the amplitude of the cycle. In the era of direct sunspot observations, there has been a long period of absence in solar activity between 1645 and 1715-with very few sunspots observed (see Fig. 2); this period is known as the Maunder minimum (Eddy 1976). There are secular trends observed in the sunspot time series with episodes of aperiodic increasing or decreasing activity which last over several cycles (scale of centuries) -with no regularity (Usoskin 2017). The quasi-periodic nature of the solar cycle, with no apparent relation between the strength of subsequent sunspot cycles makes predictions a challenging task-an exercise that has to be firmly rooted in the physics of the sunspot cycle. The subject of MHD deals with the creation of astrophysical magnetic fields and provides the theoretical basis for understanding the genesis of solar-stellar magnetism.

Material in the solar interior exists in the plasma state, wherein convective and large-scale motions can both destroy and induct magnetic fields through the dynamo 


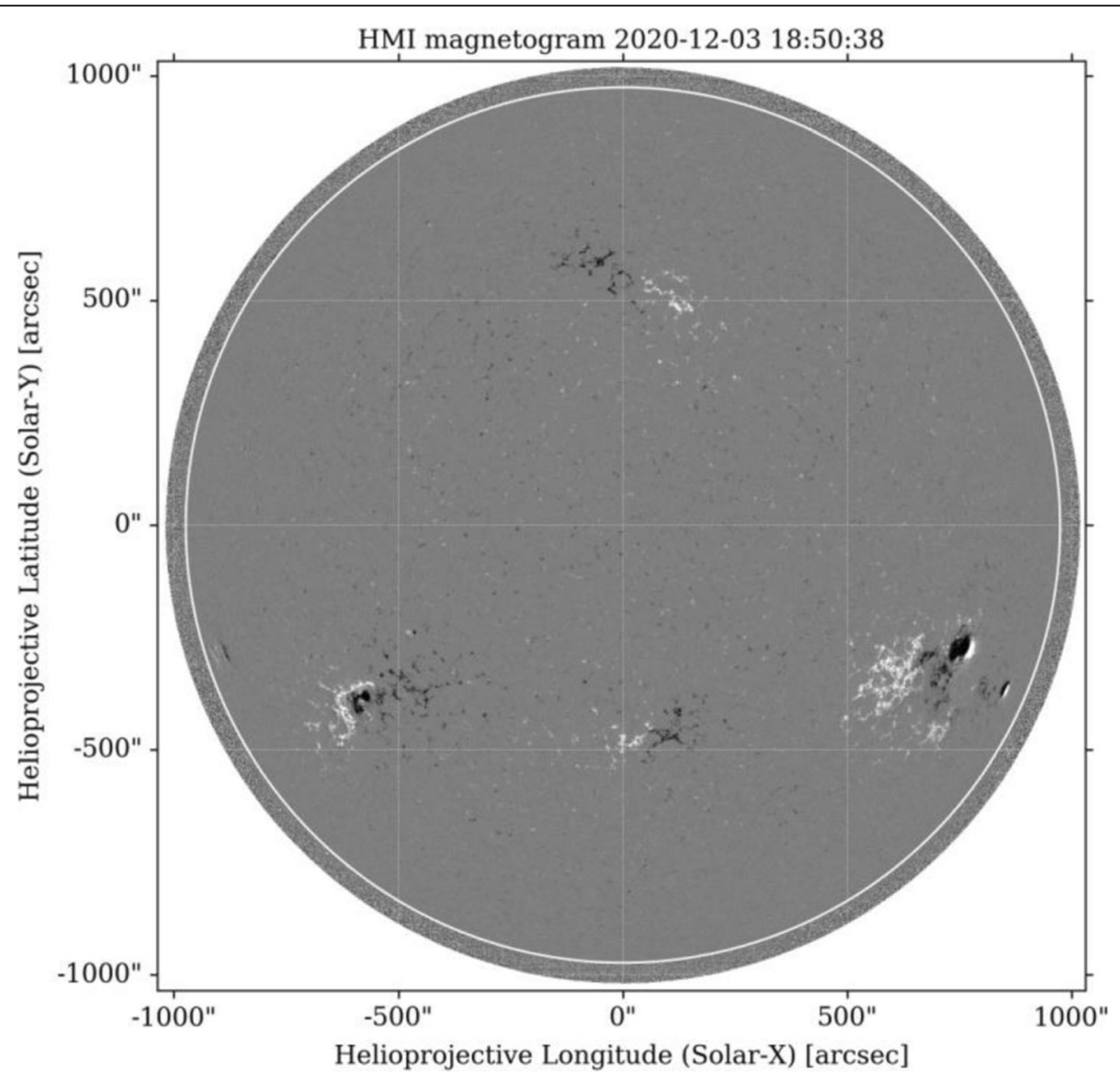

Fig. 1 A solar magnetogram shows regions of strong magnetic fields (solar active regions) on the solar surface which are typically composed of bipolar sunspot pairs (white and black regions indicate positive and negative polarity, respectively). These bipolar sunspots appear with a systematic tilt with respect to the local parallel of latitude and their relative orientation is reversed in the two hemispheres. The tilt angle that is observed within the solar active regions increases with latitude which is known as Joy's law. Sunspots and associated overlying magnetic field loops generate solar storms and modulate the radiative energy output of the Sun. They are tracers of the underlying solar dynamo mechanism. Credit: NASA HMI/SDO

mechanism (Fig. 3). When conditions are right, inductive processes result in generation of large-scale magnetic fields in stellar interiors that sustain the plethora of magnetic activity that stars display. Stars are generally born with preexisting magnetic fields that exist in the star-forming gases and rotate differentially. A preexisting poloidal (dipolar) component of the magnetic field can be stretched by differential rotation to form the toroidal component (Parker 1955a) from which buoyancy instability generates sunspots (Parker 1955b). During their buoyant rise, toroidal flux tubes are subject to the Coriolis force which imparts tilt and thus sunspots appear as bipolar pairs with a systematic tilt relative to the local East-West direction. Turbulent, helical convection in rotating systems can work on the buoyantly rising toroidal flux tubes to regenerate the poloidal field through a process known as the mean-field $\alpha$-affect (Parker 1955a). The decay and dispersal of the flux of tilted bipolar sunspot pairs, whose evolution is governed by near-surface plasma flows-such as supergranular diffusion, differential rotation, and meridional circulation-can also regenerate the large-scale dipolar field of the Sun resulting in the reversal of the old cycle polar field and imparting a new polarity; this process is widely known as the Babcock-Leighton mechanism (Babcock 1961; Leighton 1969) and is now believed to be the dominant process driving solar cycle fluctuations (Cameron and Schüssler 2015; Bhowmik and Nandy 2018). This continuous recycling of flux between the toroidal and poloidal components of the solar magnetic field is believed to sustain the solar cycle.

Modeling the full breadth of complex, non-linear interactions between plasma flows and magnetic fields in the extreme regimes of stellar interiors remains a challenging task. While full MHD, direct numerical simulations are yet to achieve realistic parameter regimes of stellar convection zones, they are beginning to display some of the characteristics of solar plasma flows and 


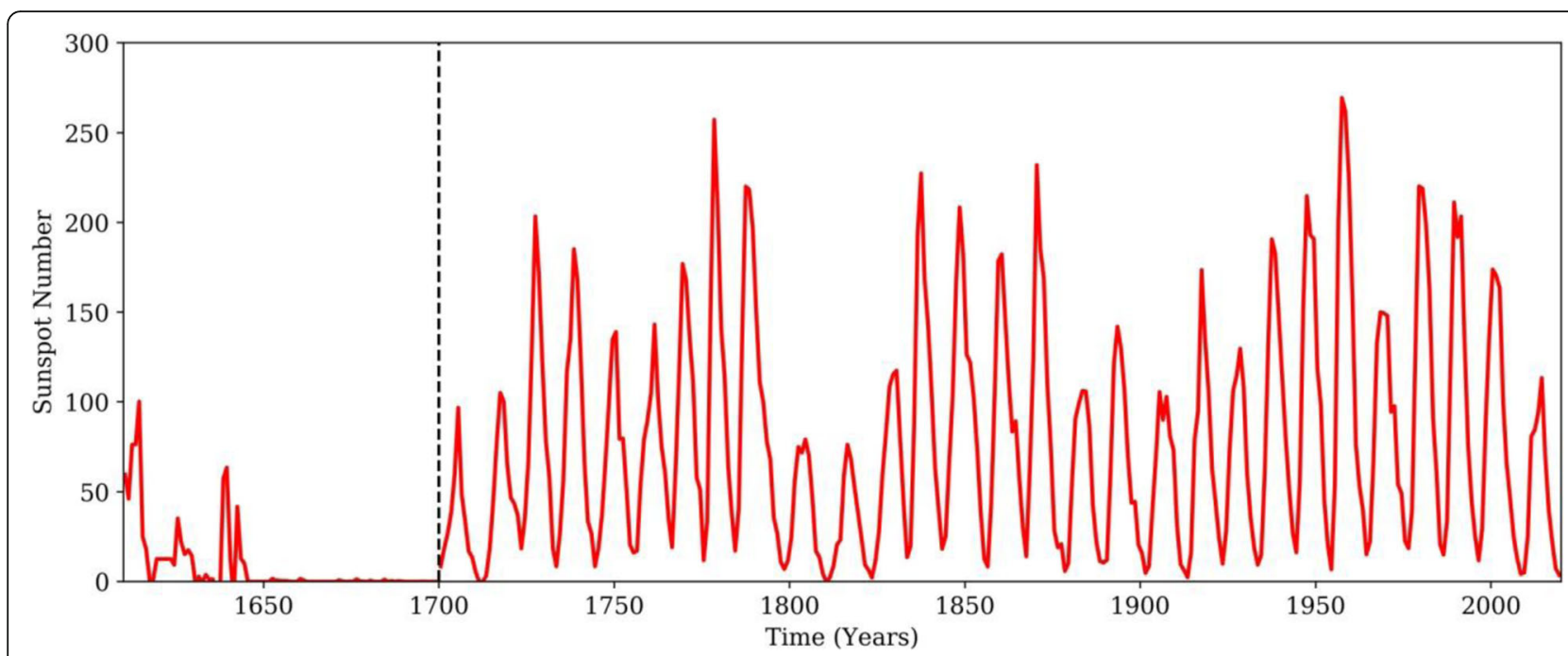

Fig. 2 The yearly mean total sunspot number time series. Observations spanning four centuries show that the number of sunspots observed on the solar surface increases and decreases in a quasi-periodic fashion with a mean period of 11 years. The plot shows the yearly mean ISN ( $v 2.0$ ) from 1700 until 2019.5. The plot 1700 (the vertical dashed line) show Group Number series (v 1.0). This is scaled by a multiplicative factor of 10 to amplify the plot until the dashed line. The amplitude of sunspot cycles varies from one cycle to another and displays secular trends that are not well-understood. A phase of very low solar activity with very few sunspot observations-known as the Maunder minimum occurred between 1645 and 1715. Solar activity reconstructions over thousands of years indicate the presence of many more extremely low or strong activity phases in the solar cycle (Usoskin 2017). Credit: SILSO, Royal Observatory of Belgium, Brussels

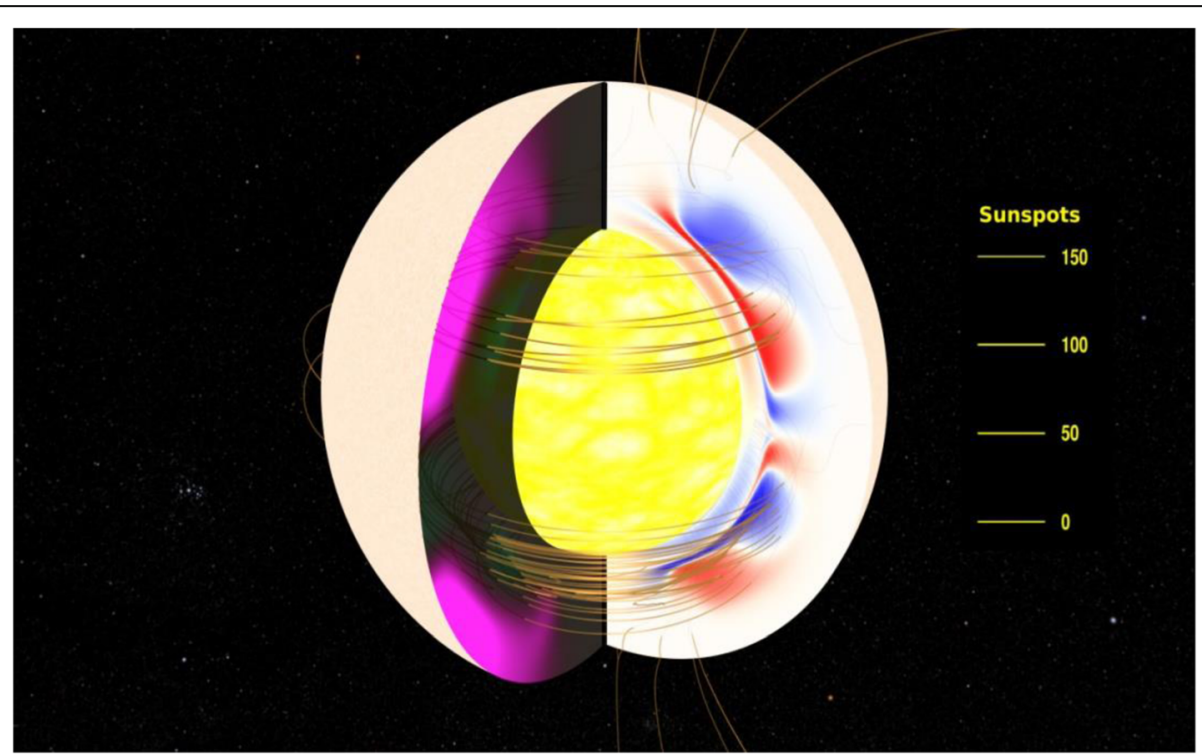

Fig. 3 A rendering of a solar dynamo simulation from Muñoz-Jaramillo et al. (2009) shows the distribution of magnetic fields within the Sun's convection zone at a phase near solar minimum. The left meridional cut depicts the poloidal component (in purple color) and the right meridional cut depicts the toroidal component (red and blue colors indicate opposite polarities). The central yellow region corresponds to the base of the convection zone. On the right, a bar plot denotes the number of sunspots on the solar surface (with yellow lines depicting different values). Since this snapshot corresponds to a solar minimum, there are no sunspots on the surface. The full movie of the simulation can be found at https://svs.gsfc.nasa.gov/3521. Credit: Tom Bridgman, NASA/Goddard Space Flight Center Scientific Visualization Studio 
complexes of magnetic activity, including cyclic reversal (Brun et al. 2004; Ghizaru et al. 2010; Nelson et al. 2013; Hotta et al. 2016; Karak and Miesch 2017; Käpylä et al. 2017). At the same time, physically inspired kinematic dynamo models based on the Babcock-Leighton ideaincreasing in sophistication and parameterization of known physics-have been developed which better reproduce the observed features of the solar cycle, such as cyclic reversal, latitudinal distribution of sunspots, and the observed phase relationship between the sunspot cycle and polar fields (Durney 1995; Choudhuri et al. 1995; Dikpati and Charbonneau 1999; Nandy and Choudhuri 2001, 2002; Nandy 2002; Guerrero and Muñoz 2004; Chatterjee et al. 2004; Guerrero et al. 2008; Muñoz-Jaramillo et al. 2009; Muñoz-Jaramillo et al. 2010; Nandy et al. 2011; Yeates and Muñoz-Jaramillo 2013; Hazra and Nandy 2016; Kumar et al. 2019). Figure 3 depicts the internal solar magnetic fields generated from such kinematic dynamo simulations (MuñozJaramillo et al. 2009; Nandy et al. 2011).

\subsection{Predicting solar activity}

While efforts to understand the solar cycle have now spanned half a century (see, e.g., the review of Charbonneau 2020), focused investigations of the physical basis of solar cycle predictions have spanned only about a decade. A scrutiny of solar cycle observations already hints that the strength of the polar field at the minimum of a cycle is a good indicator of the strength of the next cycle (Fig. 4). Indeed the more successful empirical, precursor-based cycle predictions take advantage of the observed correlation between the polar field at minimum and the next cycle amplitude (Schatten et al. 1978). For solar cycles 23 and 24, multiple different methodologies, e.g., statistical correlations based on precursors of solar activity, time-series analysis of solar cycle observations, machine learning techniques, solar surface flux transport models, and solar dynamo models, have been utilized for predictions often resulting in wide disagreements. It is beyond the scope of this manuscript to review all these predictions and the methodologies employed; for further details, readers are referred to the more exhaustive review of the literature by Pesnell (2008), Petrovay (2020), Nandy (2021) and the collection of articles catalyzed by the SEE project published in a special issue of the Journal of Atmospheric and Solar-Terrestrial Physics (Obridko and Georgieva 2018).

It is only now that we are beginning to appreciate the intricate details of how physical processes in the solar interior determine the predictability of solar cycles. We briefly summarize here the progress in our understanding of solar cycle predictions gleaned over the last decade (and the SEE project era) which has been discussed in details in Nandy (2021). First of all, theoretical arguments indicate that the Babcock-Leighton dynamo mechanism of poloidal field generation, governed by the emergence of tilted bipolar active regions and their flux dispersal by near-surface flux transport processes, is the dominant driver of poloidal field generation in the Sun (Cameron and Schüssler 2015). Observations of tilt angle distribution of sunspot groups and their empirical relationship with the amplitude of sunspot cycles also support this scenario (Dasi-Espuig et al. 2010). However, the two dynamo-based forecasts for solar cycle 24 based on assimilating the Babcock-Leighton idea (Dikpati et al. 2006; Choudhuri et al. 2007) diverged by a wide margin inviting controversy and uncertainty in the community. Subsequently, Yeates et al. (2008) demonstrated that the diverging predictions resulted from different parameterizations (advection dominated versus diffusion dominated) of the SCZ in the two models and there is nothing problematic with the basic premise of the Babcock-Leighton idea itself in terms of its usage in predictive dynamo models.

Recent studies indicate that the interplay of magnetic flux transport processes such as turbulent diffusion (Muñoz-Jaramillo et al. 2011), meridional circulation (Choudhuri et al. 1995), and turbulent pumping (Käpylä et al. 2006a, 2006b; Guerrero et al. 2008; Hazra and Nandy 2016) impact the memory and predictability of solar cycles with the predictive window being restricted to only one cycle (Karak and Nandy 2012; Hazra et al. 2020). These studies suggest that very long-term prediction beyond the following solar cycle is not possible at the previous minimum. While solar cycle amplitude variations can result from diverse physical mechanisms, both observations ((Dasi-Espuig et al. 2010) and datadriven modeling of solar activity (Bhowmik and Nandy 2018; Jiang et al. 2018) suggest that the scatter in active region tilt angle distribution is the dominant driver of cycle-to-cycle amplitude variability. In this context, anomalous active regions with tilt angles that depart strongly from Joy's law and anti-Hale regions can strongly perturb the polar field build-up process introducing an element of uncertainty in predictive models relying on the Babcock-Leighton idea (Nagy et al. 2017). However, these can be reasonably accounted for via ensemble forecasts-which imbibe reasonable uncertainty ranges in active region input properties (tilt angle, flux, and latitude of emergence), as has been done in recent predictions for solar cycle 25 (Bhowmik and Nandy 2018; Jiang et al. 2018; Labonville et al. 2019).

Physical model-based predictions for solar cycle 25 have broadly followed three approaches. In one approach, surface flux transport models (which assimilate the observed statistics of sunspot emergence) are utilized to predict the polar field in advance of the minimum; subsequently, the empirical relationship between polar 


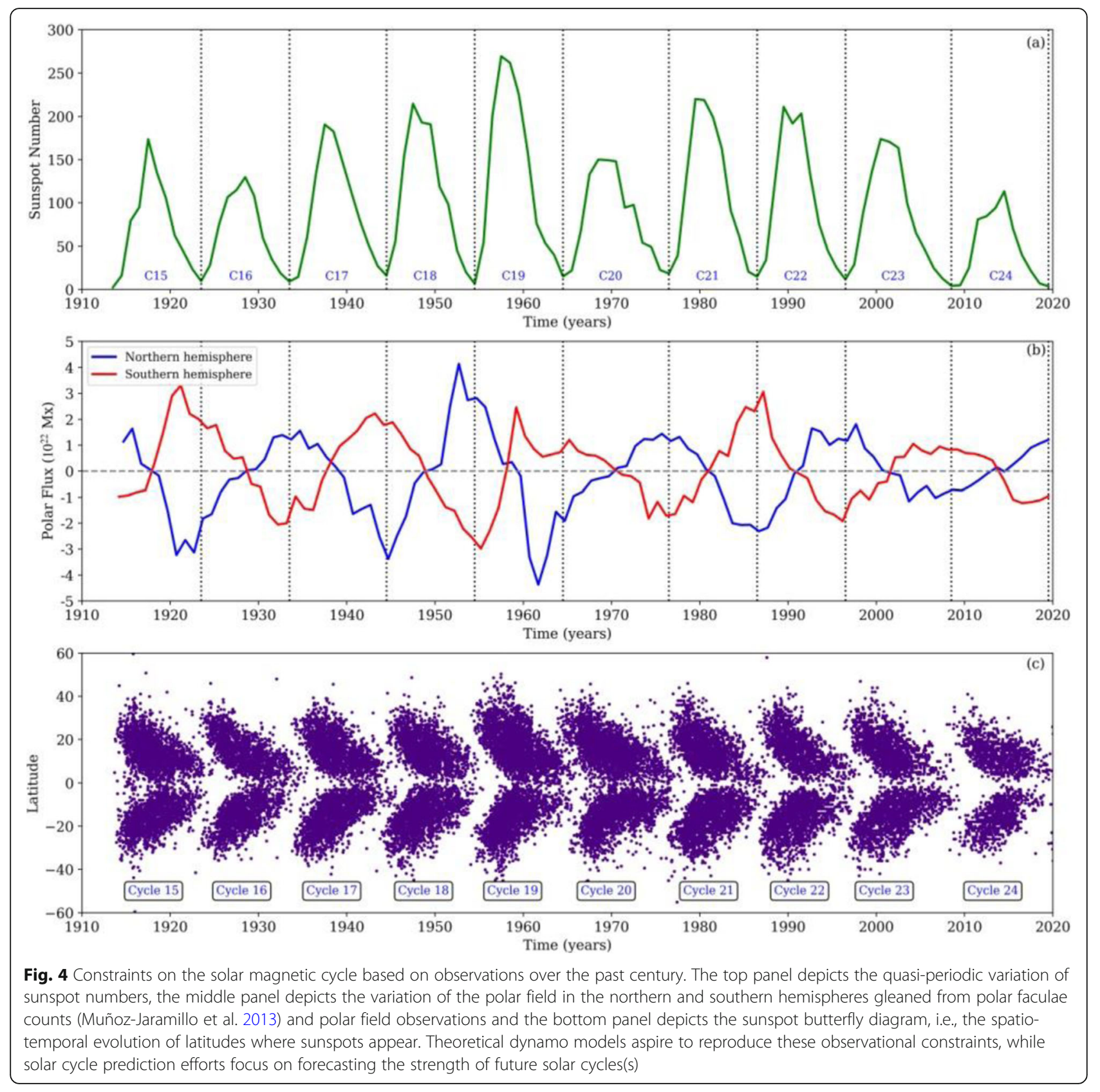

fields at minima and subsequent cycle amplitudes is used to get an indication of the future cycle (Cameron et al. 2016; Iijima et al. 2017; Upton and Hathaway 2018; Jiang et al. 2018). In another approach, data-driven solar dynamo models (assimilating the polar field observed at the minimum) are used to predict the upcoming solar cycle (e.g., used for cycle 24 predictions by Dikpati et al. 2006; Choudhuri et al. 2007). In the third, recently developed and more complete physics-based approach, data-driven models of magnetic field evolution in the solar surface and in the interior (i.e., surface flux transport and dynamo models) are coupled to generate solar cycle predictions (Bhowmik and Nandy 2018; Labonville et al. 2019). The physics-based approaches for predicting solar cycle 25 have converged and all indicate a weakmoderate cycle similar or slightly stronger than cycle 24 (see Fig. 5). The timing of the peak is estimated to be around 2024-2025. Nandy (2021) argues that this convergence in physical model-based predictions for sunspot cycle 25 is a consequence of advances in our understanding of solar cycle predictability discussed earlier.

Fuelled by the declining trend in solar cycle activity over the last several decades, there was widespread 


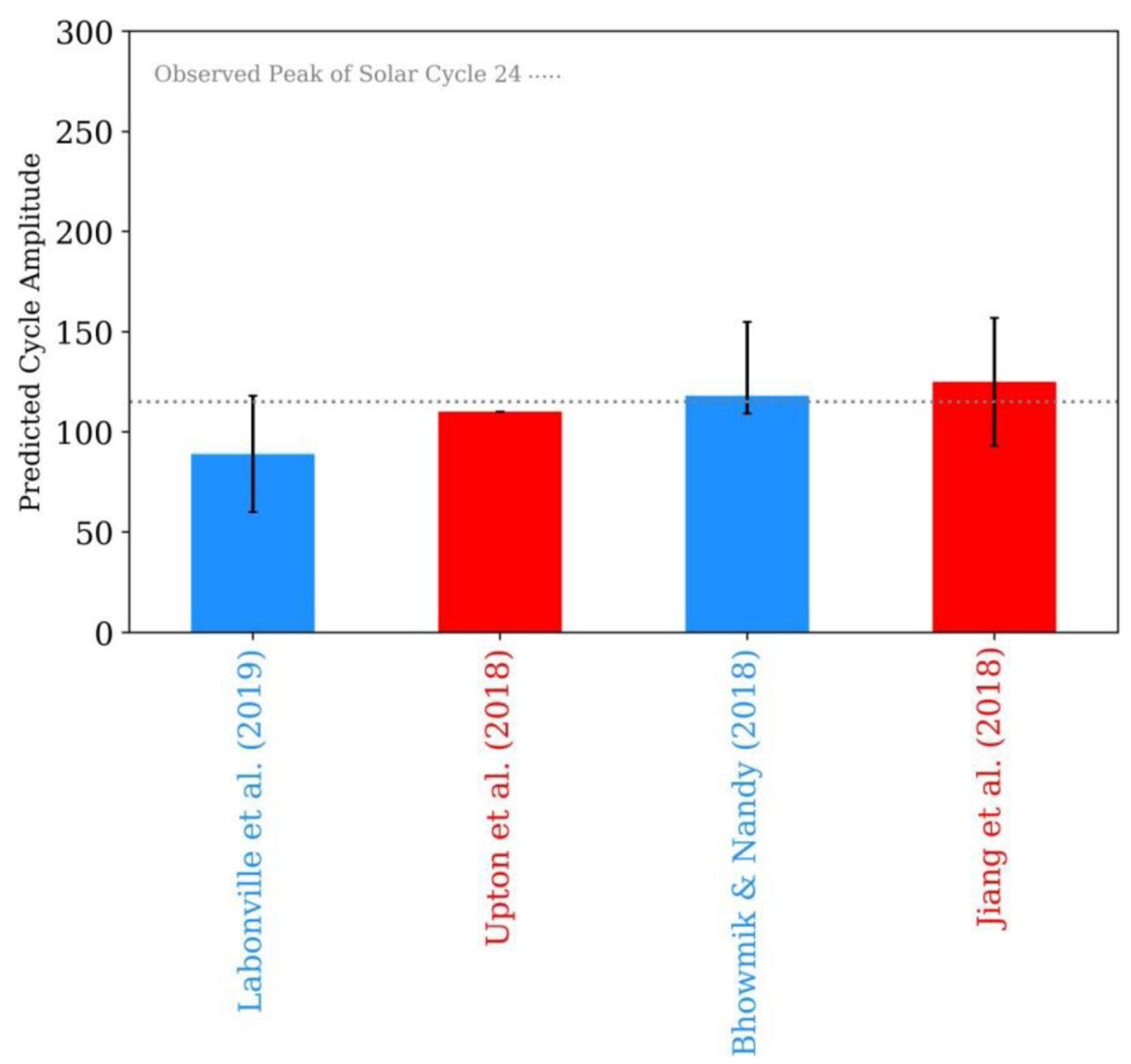

Fig. 5 A summary of physical model-based predictions for solar cycle 25 indicates that the forecasts have converged and indicates a weak cycle, similar or slightly stronger relative to the previous cycle 24. Adapted from Nandy (2021)

speculation of an impending Maunder-like grand minimum in solar activity prior to the minimum of cycle 24 (Miyahara et al. 2010). However, as demonstrated in the various physical model-based predictions (Fig. 5), there is going to be a solar cycle 25 , whose early signatures are already being observed (Nandy et al. 2020). As Nandy (2021) has argued, the current physical understanding of solar cycle predictability does not allow any indication of long-term future activity beyond cycle 25 at this point in time.

\subsection{Solar extrema: grand minima and maxima in activity}

While direct sunspot observations exist only from the early seventeenth century, indirect proxies of solar activity based on cosmogenic isotopes have been utilized for reconstructing past solar activity (Fig. 6). Such reconstructions indicate the presence of multiple grand maxima and minima in activity, when solar activity levels either exceed or fall below typical activity levels over a few cycles, the most recent and striking minimum in activity being the Maunder minimum (Usoskin et al. 2014). It is expected that during high activity levels the frequency of solar storms would be higher, while during activity minima, there would be hardly any solar flares or CMEs-thereby resulting in strong and weak geospace perturbations (Gopalswamy 2016) during activity maxima and minima, respectively. On the other hand, cosmic ray fluxes in the heliosphere are at extreme levels during low solar activity phases. Heliospheric conditions therefore depend on solar extrema. There is emerging (although not yet mature) understanding that very longterm solar activity levels modulate the Earth's climate; however, this relationship is muddled by the unprecedented anthropogenic forcing in the recent past (Haigh 2007). Solar cycle variability during the maunder minimum is studied extensively using all available sunspot observations in Usoskin et al. (2015).

While there have been some attempts at identifying long-term periodicities such as the centennial scale Gleissberg cycle in the sunspot record, this is more likely a modulation of the envelope of solar activity rather than any long-term periodic behavior (Usoskin 2017). Statistics of grand solar maxima and minima in activitywhich are widely separated intermittent occurrencesshow that there is no regularity to these events, with their distribution being governed by a stochastic and 


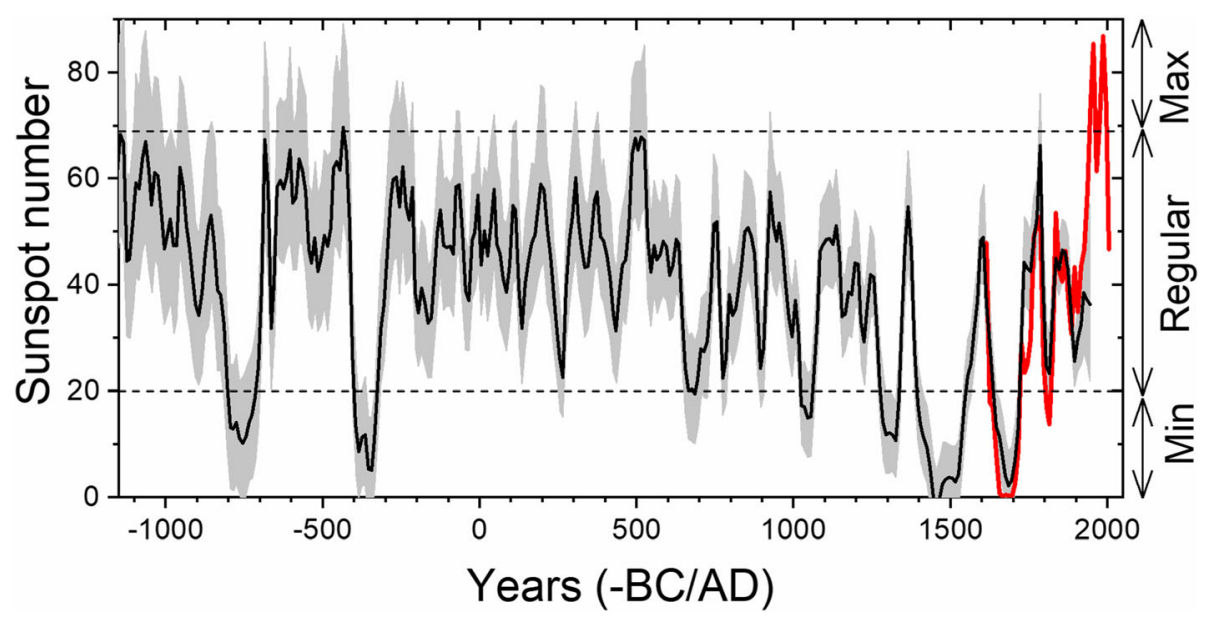

Fig. 6 Solar activity reconstruction over the past 3000 years shows a few extreme activity phases during which the sunspot cycle amplitudes persistently exceed (grand maxima) or fall below (grand minima) the typical activity levels (denoted within the two dashed lines). Following the Maunder minimum (1645-1715) and Dalton minimum (1790-1830), a growth in general activity levels resulted in the modern maximum. Solar cycle 24 however was very weak and cycle 25 is expected to be a below average cycle too. Further in the past, other grand maxima and minima episodes are observed indicating extreme activity levels have occurred over the recent history with no apparent regularity. Adapted from Usoskin et al. (2014)

(or) chaotic process (Mininni et al. 2002; Usoskin et al. 2007). Theoretical arguments underscore that the grand minima phase is a special state of the solar dynamo, distinct from other normal or high activity phases (see Tripathi et al. 2021 and references therein). There are some indications from solar activity proxies that weak magnetic cycles in the large-scale solar magnetic field persisted along with few sunspot appearances during the Maunder minimum (Mursula et al. 2001; Miyahara et al. 2004). There are also hints from observations that strong hemispheric asymmetry in solar activity (Temmer et al. 2002) is associated with grand minima episodes with sunspots predominantly erupting in one hemispherewith consequent hemispheric decoupling in activity (Usoskin 2017). Studies indicate that the nature of the coupling between dipolar and quadrupolar modes of the solar dynamo mechanism (DeRosa, Brun and Hoeksema 2012) and the parity of the solar cycle may be connected to activity fluctuations (Weiss and Tobias 2016; Beer et al. 2017; Obridko et al. 2020).

Long-term fluctuations in solar activity, including secular trends and extreme activity levels, are theoretically understood to originate from stochastic fluctuations in driving parameters of the solar dynamo mechanism (Passos et al. 2014), non-linear feedback mechanisms between magnetic fields, convection, and plasma flows (Tobias 2002; Wilmot-Smith et al. 2005; Muñoz-Jaramillo et al. 2011; Weiss and Tobias 2016), time-delay dynamics (Wilmot-Smith et al. 2006; Jouve et al. 2010), or a combination of these effects Hazra et al. 2014; see Fig. 7). Long-term observations of internal plasma flow variations such as torsional oscillations in the differential rotation and meridional circulation-which are indicative of non-linear feedback of the magnetic field on the flows-are limited to about two solar cycles (Lekshmi et al. 2018; Lekshmi et al. 2019). While they reveal interesting physics, they are not long enough for plasma flow variation-driven long-term dynamo simulations. Therefore, the latter focus on a variety of theoretically expected physical mechanisms to explore very long-term solar activity fluctuations. For more comprehensive reviews of various mechanisms that may induce long-term modulation in solar activity, interested readers are referred elsewhere (Tobias 2002; Charbonneau (2020).

Interestingly, recent, better formulated approaches to model long-term solar activity variations, including entry and exit from grand minima episodes, suggest that a weak, mean-field $\alpha$-effect is necessary in conjunction with the large-scale Babcock-Leighton mechanism for recovering solar-stellar activity cycles following a prolonged grand minima episode (Hazra et al. 2014; Passos et al. 2014). While such long-term simulations are computationally expensive, 2D kinematic simulations show that hemispheric decoupling in activity may occur during low activity phases with one hemisphere remaining quiescent while the other hemisphere continues to show sunspot activity (Passos et al. 2014; Fig. 8). Recent solar dynamo models have also shown that long-term modulation and such hemispheric asymmetry may eventually lead to parity shifts (between dipolar and quadrupolar modes) in the solar magnetic cycle (Hazra and Nandy 2019). Such studies are only now beginning to reveal how the stochastic nature and intricate non-linear physics of MHD interactions in stellar convection zones can 


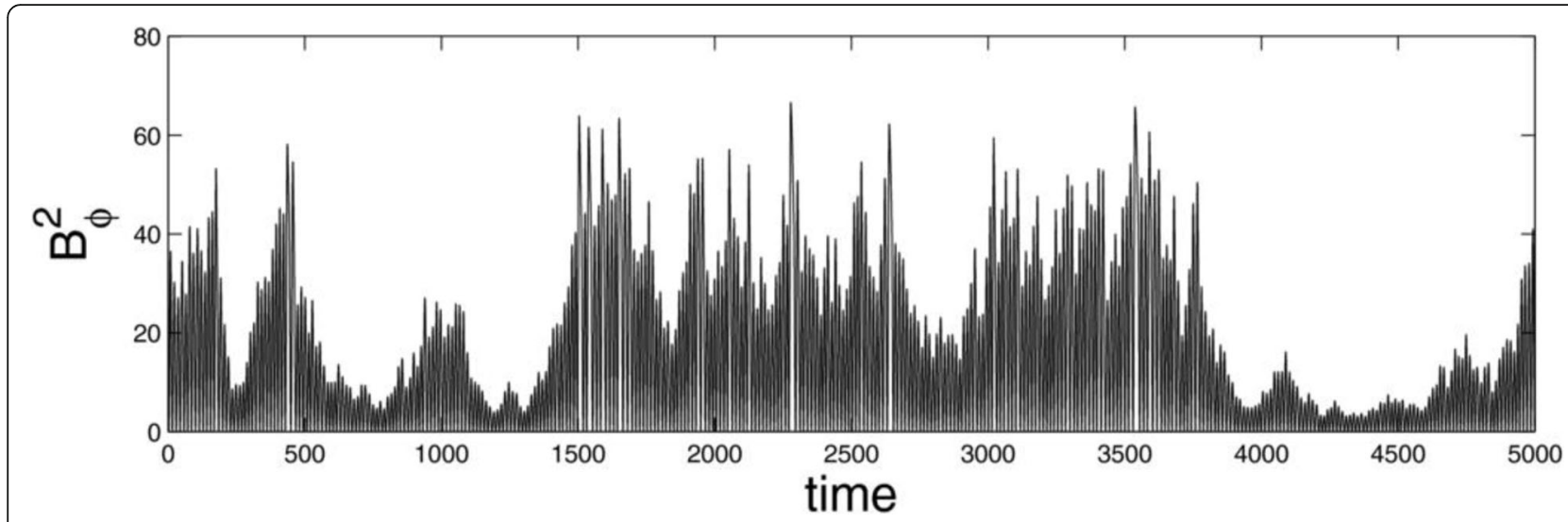

Fig. 7 Evolution of magnetic energy proxy versus time in a reduced, time-delay solar dynamo model with non-linearity and stochastic forcing (Hazra et al. 2014). These very long-term simulations recover the existence of grand maxima and minima phases which are intermittently distributed over the course of the simulations

lead to long-term symmetry changes and activity modulation in the solar dynamo mechanism (Käpylä et al. 2016; Raynaud and Tobias 2016).

\subsection{Solar forcing of the heliosphere}

As discussed earlier, the activity of the Sun changes both during the course of a cycle and over a longer term due to modulation of the solar dynamo mechanism over centennial to millennial timescales. This variation induces short- and long-term variations in space environmental conditions such as radiation, energetic particle flux, and heliospheric magnetic fields. Planets are impacted by these variable space environmental conditions governed by solar activity, thus sharing a causal relationship with the Sun.

Sunspots, when they first appear, tend to reduce the overall solar radiation output due to their inhibitory effect on convective energy transfer to the surface. However, within a solar rotational timescale, sunspot decay and their associated network regions-faculae and

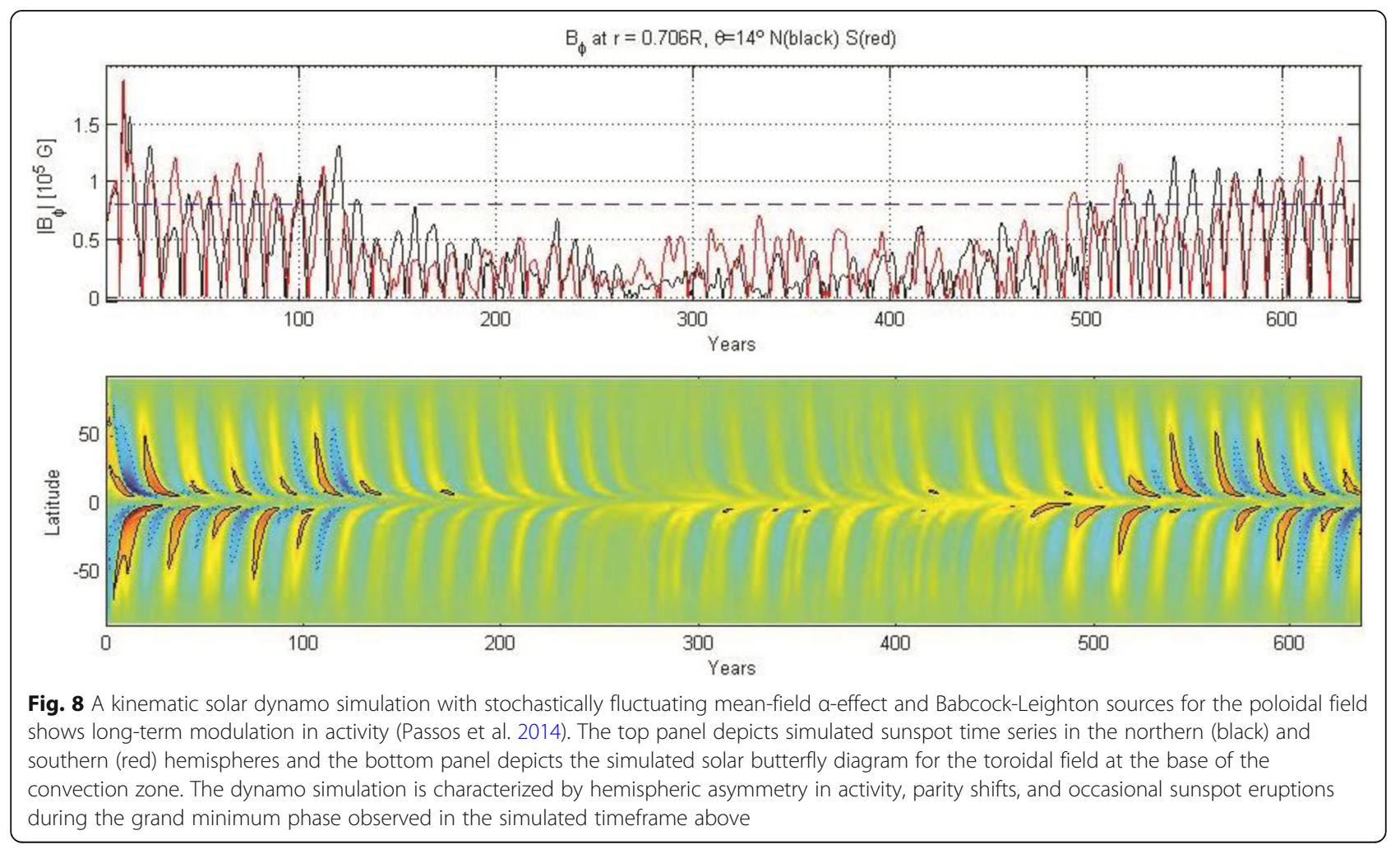


plage-result in an increase in the total solar radiative output (Krivova et al. 2007; Yeo et al. 2020). This results in an overall positive correlation between solar magnetic cycle and solar radiative output over decadal and longer timescales. This is indeed observed in satellite-based measurements of the Total Solar Irradiance (TSI; Fig. 9) - the solar energy incident per unit area per unit time at $1 \mathrm{AU}$. Note that radiation fluxes in (individual) higher frequencies (spectral irradiance) may vary much more over the solar cycle. Given that the radiation flux of the Sun is the primary natural energy source for heating of planetary atmospheres and driving planetary climates, there is strong interest in understanding how the past activity of the Sun may have influenced our climate. Efforts have been made for long-term reconstruction of TSI variations over millennia based on physics-inspired empirical modeling (Vieira et al. 2011; Yeo, Krivova and Solanki 2014; Wu et al. 2018). Such efforts form SunClimate relationship studies that seek to understand the impact of long-term solar radiative variability on planetary atmospheres (Haigh 2007) and also provide inputs to the assessment reports of the Intergovernmental Panel on Climate Change (IPCC Report 2013; https://www. ipcc.ch/report/ar5/wg1/).

The emergence, evolution, and dispersal of solar magnetic fields on the solar surface govern the evolution of coronal and heliospheric magnetic fields, the latter mediated by the solar wind. The heliospheric open magnetic flux plays an important role in determining the state of the heliosphere. The solar open flux can be reconstructed based on established relationships between solar surface magnetic activity and coronal fields at the source surface-where the solar wind becomes dynamically important and carries the solar magnetic field throughout the solar system. Ideas about solar wind and near-Earth heliospheric magnetic fieldvariations over century scale are now available (Owens et al. 2016; Owens et al. 2017). Based on an understanding of solar magnetic field variations, long-term reconstructions of the solar open flux are now possible (McCracken 2007; Lockwood et al. 2014).

The heliospheric (solar) open magnetic flux is the key contributor to variations in the cosmic ray flux (Fig. 10). Thereby, this provides a causal connection between solar activity and galactic cosmic ray intensity at Earth; higher solar activity and open flux levels result in lower cosmic ray flux. Cosmic ray interactions with the Earth's atmosphere generate radionuclides (e.g., ${ }^{10} \mathrm{Be}$ and ${ }^{14} \mathrm{C}$ ) - which make their way in to tree rings and ice sheets. Therefore, measurements of radionuclide abundances and inversion techniques provide an ingenious methodology to reconstruct solar activity (Usoskin 2017); it is important to
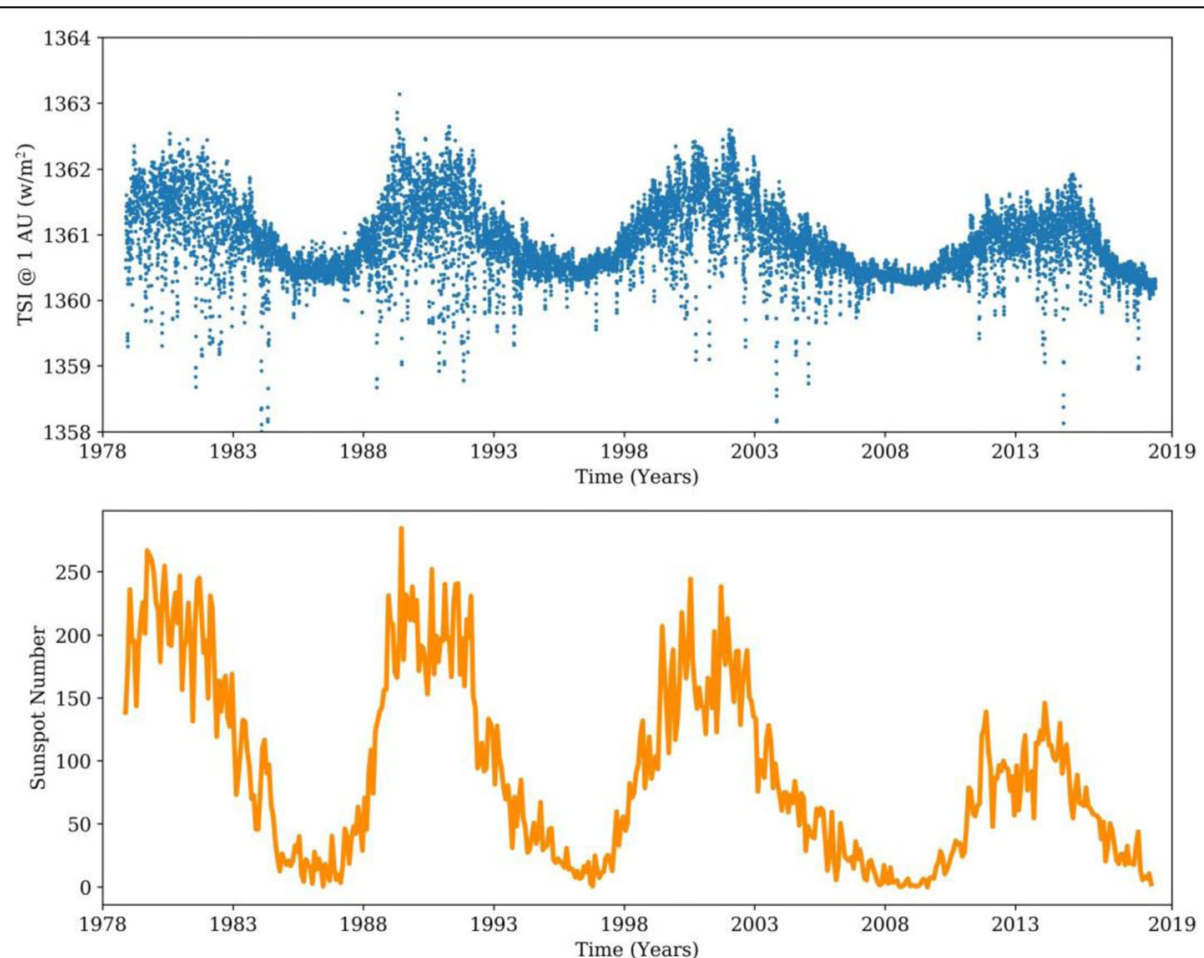

Fig. 9 Comparison of total solar irradiance (TSI) variations (top panel) with the sunspot time series (bottom panel). The TSI varies in phase with the solar cycle indicating that during phases of very strong activity, the total radiation output-and hence radiative forcing of the heliosphere-is enhanced. The composite TSI time series data is acquired from PMOD/WRC (Fröhlich 2006) 


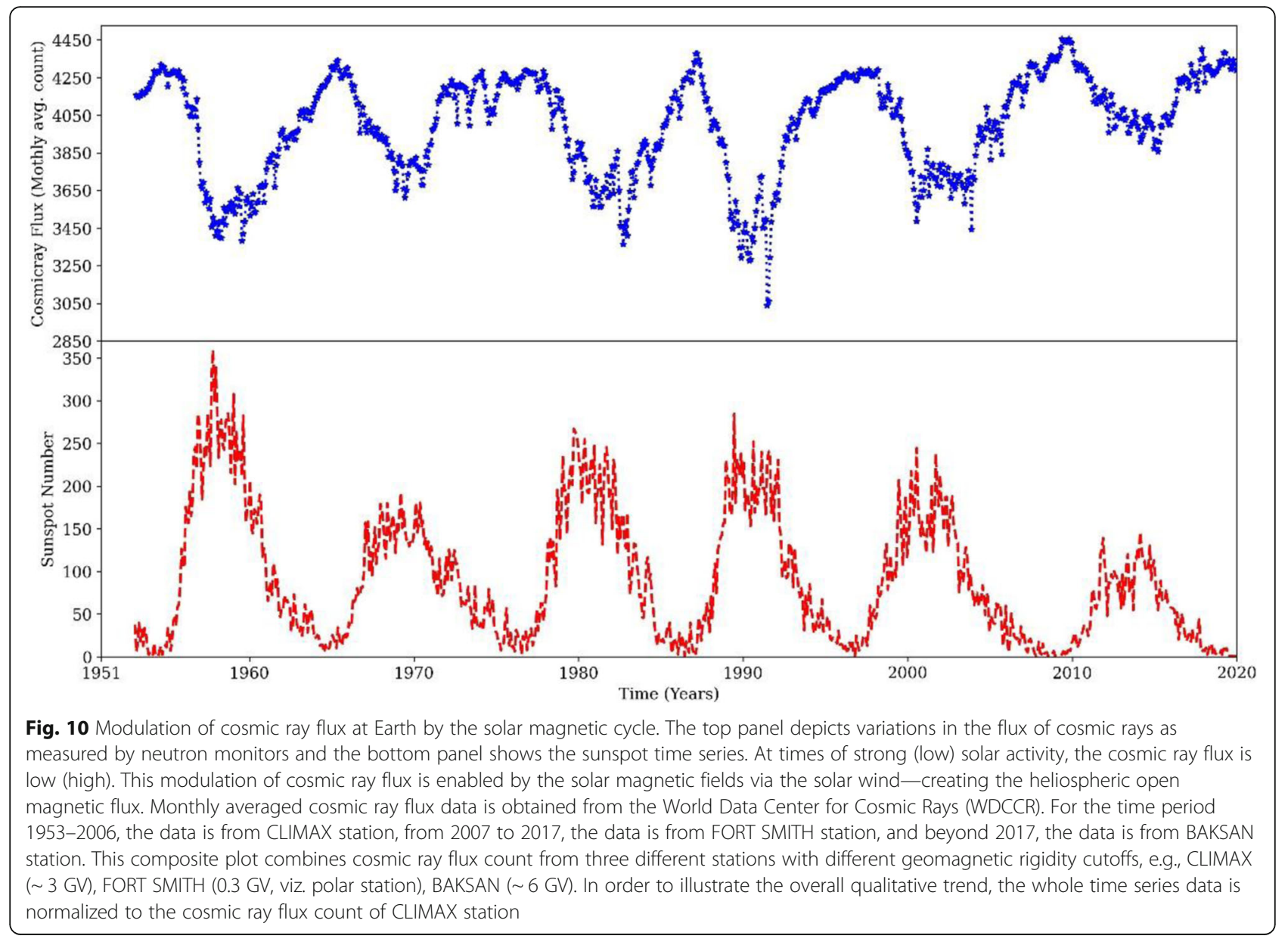

note that such reconstructions are limited to about 1012 millennia.

Recent investigations also show other unique roles of the heliospheric open flux; interactions mediated via magnetic reconnection between propagating magnetic storms from the Sun and the open flux can in fact reduce the geo-effectiveness of solar storms (Pal et al. 2020). Indeed, magnetic connectivity from the Sun and throughout the solar system provides a causal basis for a diversity of planetary impacts of the activity of our parent star; the intricacies of these causal connections sustained over planetary lifetimes lead to other interesting phenomena discussed in the next section.

\section{Evolution of solar-stellar activity: implications for planets}

The solar system teaches us that stellar activity influences planets in profound ways. Magnetohydrodynamic processes-similar to what is in evidence for the Sunoperate in the interior of other solar-like stars. However, given that different stars have different rotation rates and differential rotation-which is believed to be a function of stellar age-the exact nature of the dynamo mechanism as well as the stellar activity is expected to vary across stars and over the lifetime of an individual star (Nandy 2004; Brun et al. 2015; Guerrero et al. 2013, 2019). Thus stellar observations and dynamo modeling of solar-like stars (or solar twins as they are known) and solar-stellar connection studies are expected to generate better constraints on the solar dynamo mechanism on the one hand and, on the other hand, provide an indication of magnetic activity evolution over the lifetime of the Sun (Nandy and Martens 2007). In turn, the expected radiative flux, solar wind, and solar storm frequency and severity are also expected to vary in the lifetime of the Sun. This stellar variability is expected to influence planetary space environments over the evolutionary lifetime of star planet systems, studies of which have implications for planetary habitability in the solar system and beyond.

\subsection{Star-planet interactions: impact on planetary atmospheres}

Stellar activity influences planetary environments and atmospheres in ways that may determine the habitability of planets (Lammer et al. 2012). For far-out planets, it is 


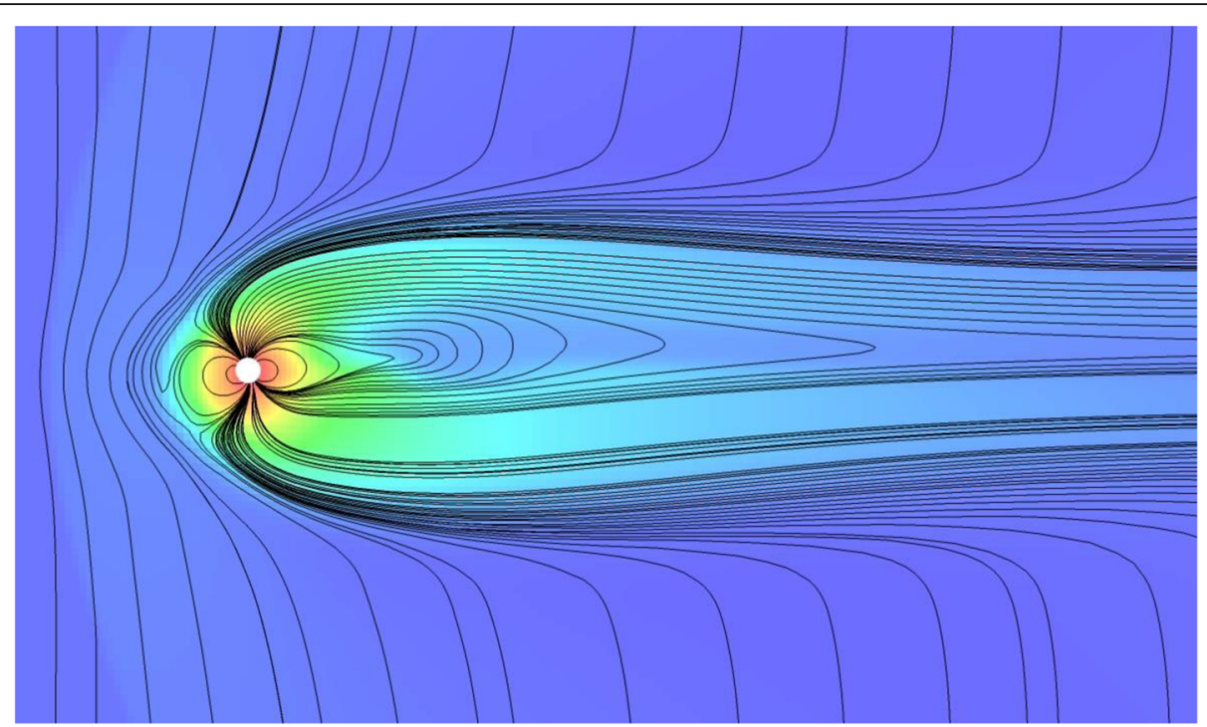

Fig. 11 Simulation of the interactions of solar wind with the Earth's magnetosphere. A plane perpendicular to the solar ecliptic (xz plane) is depicted. Earth is denoted by the white circle. Nearly vertical black contours on the left of the image indicate the solar wind magnetic field, whereas curved black lines_one or two ends of which are rooted on Earth_indicate the Earth's magnetospheric field following a dynamic equilibrium with the solar wind. Magnetic reconnection and other fluid dynamical and MHD processes play critical roles in determining the impact of stellar magnetized winds and storms on planetary magnetospheres and atmospheres. These processes can lead to atmospheric mass loss or injection (via plasma flows through reconnected field lines), and over long timescales can influence planetary habitability. The above simulation was performed using CESSI-SPIM (Das et al. 2019)

typically the activity of the star which has a one-sided impact on planetary atmospheres and evolution (Fig. 11). However, for close-in star-planet systems, wherein the planet and star's magnetospheres can dynamically interact or wherein the planet imparts sufficient tidal forces to influence stellar flows, the influence may work either way (Strugarek 2016; Strugarek et al. 2014, 2019). The environment of some planet hosting stars, e.g., Mdwarfs, would be extreme (Cohen et al. 2014), plausibly precluding the possibility of life. Many Jupiter-like large planets with such extreme environments within close proximity to host stars have been detected and are known as Hot Jupiters. Here, we confine ourselves to discussing Star-Planet Interactions (SPI) in the context of Earth-like (far-out) planetary or exoplanetary systems which are more likely to host life rather than (plausibly uninhabitable) close-in Hot Jupiter-like planets.

Primary mechanisms of SPI include but are not limited to radiation-driven sputtering processes that influence planetary atmospheric escape (Gronoff et al. 2020), radiative forcing of atmospheres (Haigh 2007), magnetic storms, and stellar wind impacts (Das et al. 2019). It is believed that the existence of a magnetosphere is useful for the protection of planetary atmospheres against solar-stellar wind and storm impact. Mars offers an interesting case study in this respect. It is believed that at about 1.5 billion years age of the solar system, when the Sun was much more magnetically active, Mars had an active dynamo; the resulting Martian magnetosphere shielded its atmosphere from the relatively strong young Sun. Mars is thought to have had a warm moist atmosphere at this point in time which could have been suitable for hosting life. Subsequently, however, cooling of Mar's core resulted in the loss of the Martian dynamo and global magnetosphere. Following sustained impact of solar radiation-driven sputtering processes, solar magnetized storms and winds, the atmosphere of Mars likely eroded away (Basak and Nandy 2021), impacting conditions for habitability (at least on or near the surface). Modeling such interactions leading atmospheric mass losses at the global level is a challenging task and attempts to do this are throwing up hitherto unanticipated nuances regarding how stellar magnetized winds interact with planetary atmospheres with or without magnetospheres or weak dipolar fields (Das et al. 2019; Sakata et al. 2020, Basak and Nandy 2021).

Studies of stellar wind environment in other astrospheres are currently being undertaken; e.g., modeling indicates that the Proxima Centauri system may have Earth-like solar wind conditions (Alvarado-Gómez et al. 2020). Search for signatures of SPI are also beginning. However, such studies are typically limited to hot Jupiter-like planets close to the host star-where SPI signatures may be detectable (Poppenhaeger et al. 2011; Strugarek et al. 2019). We anticipate that the coming decade would be an exciting era for SPI explorations, 
primarily driven by the quest to understand habitability in exoworlds.

\subsection{Solar-stellar activity over planetary evolutionary timescales}

The magnetic activity of stars evolves with age; this evolution is driven by changes in properties of the stellar convection zone and differential rotation rate and results in changes in the dynamo output, stellar surface magnetic field distribution, topology of coronal magnetic fields, frequency and strength of stellar flares, and stellar wind output. Angular momentum losses through magnetically driven stellar winds and stellar spin-down in fact reduce the efficiency of the solar dynamo mechanism with time by increasing the Rossby number (i.e., reducing the dynamo number; Nandy 2004; Ahuir et al. 2020). The magnetic output of a solar-like star therefore reduces with age (Nandy and Martens 2007; Vidotto et al. 2014); see, e.g., Fig. 12. There has been suggestions that at about stellar mid-life there is a breakdown in stellar gyrochronology relations (between rotation rate and age) in a solar-like star (Van Saders et al. 2016) and that the Sun is much less active than a typical solar-like star (Reinhold et al. 2020a). This has spurred a debate in the solar-stellar community on whether the Sun's activity is special, and whether the Sun is about to transition to a magnetically inactive future (Metcalfe and van Saders 2020; Reinhold et al. 2020b). A recent study indicates that a subcritical magnetic dynamo in the current
Sun, which allows both normal activity phases and grand minima activity phases to be accessed by the dynamo mechanism, can self-consistently explain the breakdown of gyrochronology relations and the co-existence of normal and grand minima states (Tripathi et al. 2021).

Given that the younger Sun was much more magnetically active, understanding this early solar system environment and the changing environment of planet Earth through the history of the solar system has the potential to inform studies of planetary habitability in the solar system (Guinan and Engle 2007). It is expected that these ideas would hold in other astrospheres; studies also indicate that the evolution of stellar activity influences the extent and properties of habitable zones (Gallet et al. 2017) within astrospheres.

The above motivation has spurred many investigations to understand the evolution of the activity of a solar-like star with age. Given that the solar energy output is the primary external source for driving planetary atmospheres and climate, reconstruction of solar irradiance over the lifetime of the Sun has been undertaken (Shapiro et al. 2020). Studies have explored how the solar wind may have changed over the lifetime of the Sun and solar-like stars (Ó Fionnagáin and Vidotto 2018; Ó Fionnagáin et al. 2019). The expected evolution of galactic cosmic ray flux modulated by the evolving solar activity has been modelled (Rodgers-Lee et al. 2020). The possible impact of a variable solar wind and consequent evolution of the Earth's magnetosphere over the solar

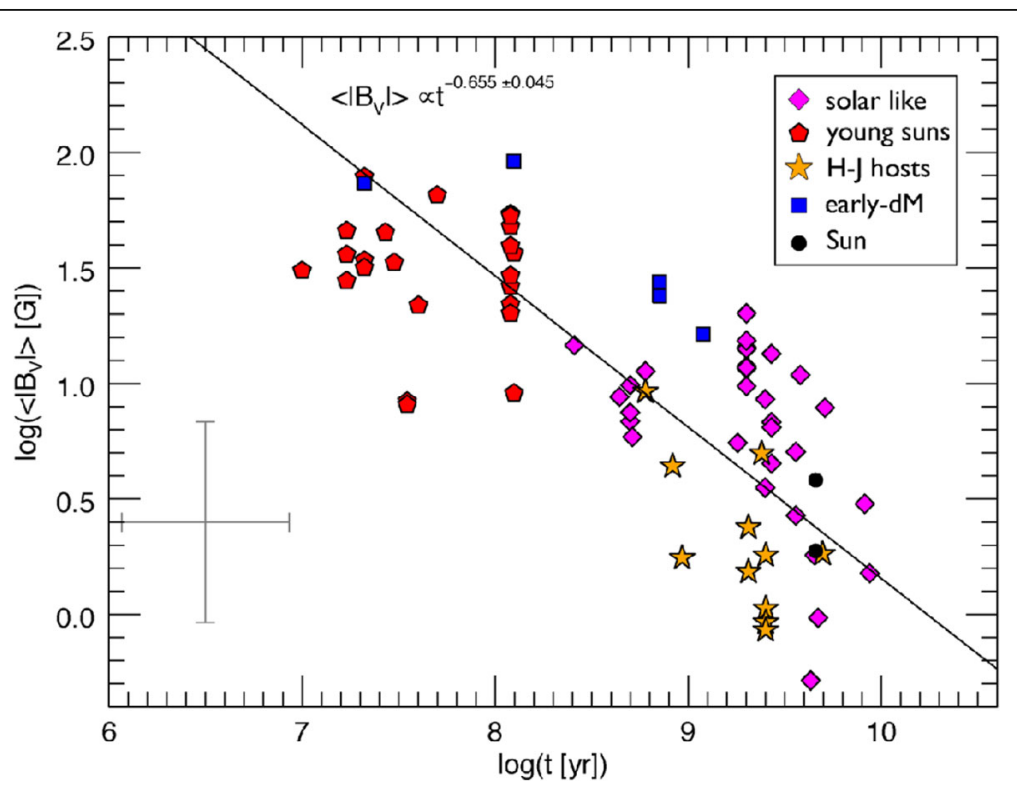

Fig. 12 Evolution of stellar magnetic activity with age (reproduced from Vidotto et al. 2014). The large-scale magnetic field is measured in this sample of stars utilizing the Zeeman Doppler Imaging technique and shows a power law dependence between magnetic activity (y-axis) and age (x-axis). These observations of stronger magnetic output at young stellar ages suggest that the environment of the early, young Sun was relatively extreme, with more energetic storms, winds, and radiative output creating an intense space environment for Earth and other solar system planets 
main sequence lifetime has been studied (Carolan et al. 2019). These studies are breaking new ground in deciphering the evolutionary history of the Sun-Earth system and are a window to star planet interactions and coupled evolution in other exoplanetary systems.

\subsection{Extreme solar storms: lessons from stellar superflares}

Extremely strong solar-stellar super storms can in principle wreak havoc on planetary atmospheres. What can be quantified as an extreme solar event, whether it be a flare or a CME? Depending on the X-ray energy output, the flares are classified into three major categories $\mathrm{X}, \mathrm{M}$, and $\mathrm{C}$ with energy budget ranging from $10^{-2}$ to $10^{-6} \mathrm{~W} \cdot \mathrm{m}^{-2}$. The bolometric energy (the energy emitted across the whole electromagnetic spectrum) in an X100 class flare is $10^{33}$ erg (see, e.g., Benz 2017). Flares with bolometric energies exceeding $10^{33}$ erg may be considered as superflares. A study in a sample of diverse classes of young stars indicated the possibility of superflares (Wolk et al. 2005); but how about stars of solarage? A subsequent study in a wider sample of stars (Fig. 13), including many solar-like stars, showed that faster rotating stars are statistically more likely to produce stellar superflares with energies as high as $10^{36} \mathrm{erg}$ with a higher frequency (Maehara et al. 2012; Shibayama et al. 2013; Notsu et al. 2013). This series of studies concluded that superflares with energies $\sim 10^{34}$ erg are possible in solar-age stars (Notsu et al. 2019) once every 6000 years (see Okamoto et al. 2021 for the most recent update).

The Carrington flare event (1859) is widely considered to be the most extreme flare ever recorded by humans; however, a solar superflare with energies close to $10^{36}$ erg has never been observed. Recently, investigations of a meteorite sample indicated that a strong superflare had occurred when the Sun was very young, only about 500 million years old (Mishra and Marhas 2019). Other studies, however, assert that the chances of these super events occurring in the current Sun are extremely low (Aulanier et al. 2013); it is also not obvious that all super events would necessarily have an extreme geomagnetic impact (Schmieder 2018). Nonetheless, the mere possibility of the current Sun launching a superflare-however low the frequency of such flares might be-merits further investigations on their likelihood and possible geospace impacts.

Flares are often associated with CMEs which are another manifestation of an energetic solar event. CMEs carry vast amounts of magnetized plasma and when their orientation is right can cause significant geospace impact and atmospheric mass loss. The fastest CME (therefore with high dynamic energy) in the space era was observed on November 10, 2004, at 02:26 UT which has a speed of $3387 \mathrm{~km} / \mathrm{s}$; there are typically very few exceeding speeds of $3000 \mathrm{~km} / \mathrm{s}$ (Gopalswamy 2016). From the statistics of CME speed distribution, estimates suggest that once in 100 years a CME event may occur with a CME speed exceeding $3800 \mathrm{~km} / \mathrm{s}$, and once in 1000 years a CME event with speed exceeding $4700 \mathrm{~km} / \mathrm{s}$ may occur (Gopalswamy 2017). These CMEs have energies ranging from $10^{33} \mathrm{erg}$ to $10^{34} \mathrm{erg}$. With favorable magnetic field orientation (southward directed zcomponent of magnetic field) and angle of impact, such CMEs could trigger devastating ionospheric and geomagnetic storms.

The energy for driving solar flares and CME events is eventually drawn from the free magnetic energy available in active region associated coronal magnetic structures or magnetic reconnection events. Thus, the amount of magnetic field output, and the possibility of super active regions produced from a very active dynamo and largescale flux tube instability increases the chances of solar super events (Schmieder 2018). It is no wonder then that younger stars with more active magnetic dynamos have a propensity of generating superflares, extreme CMEs, and strong coronal X-ray emission (Nandy and Martens 2007). What the impact of these was on geospace and other planetary atmospheres through Earth's history is an intriguing topic of future investigations.

\section{The Faint Young Sun Paradox}

While much (necessary) attention is being paid to the possible influence of the Sun on global climate variation in the last millennium, there is an even more astounding problem in the mismatch between solar luminosity and terrestrial climate in the first several billions of years of the Earth's existence, an issue known as the Faint Young Sun Paradox (FYSP; Kasting et al. 1988). In brief, the paradox is this: The geological and biological record support that the Earth's biosphere was considerably warmer than currently during the origin of life on Earth and for several billions of years thereafter (e.g., Kasting 1989). Yet, stellar evolution calculations support the Sun reaching the Zero Age Mean Sequence (ZAMS) at about $70 \%$ of its present luminosity, and linearly increasing in time up to its current level. Climate models predict a "Snowball Earth;" for such a low solar constant, unless the greenhouse effect were much stronger than what it is now (see Feulner 2012). However, there is no geological evidence for a hugely increased presence of greenhouse gasses in the early atmosphere (Kasting 2004).

Expanding our view to the evolution of stars in general, this aspect of stellar evolution theory implies that the habitable zone around stars moves outward as the star matures, due to the gradual but significant increase of stellar luminosity over the star's lifetime. Hence, ceteris paribus, planets that start out with conditions favorable for life, liquid water in particular, heat up as 


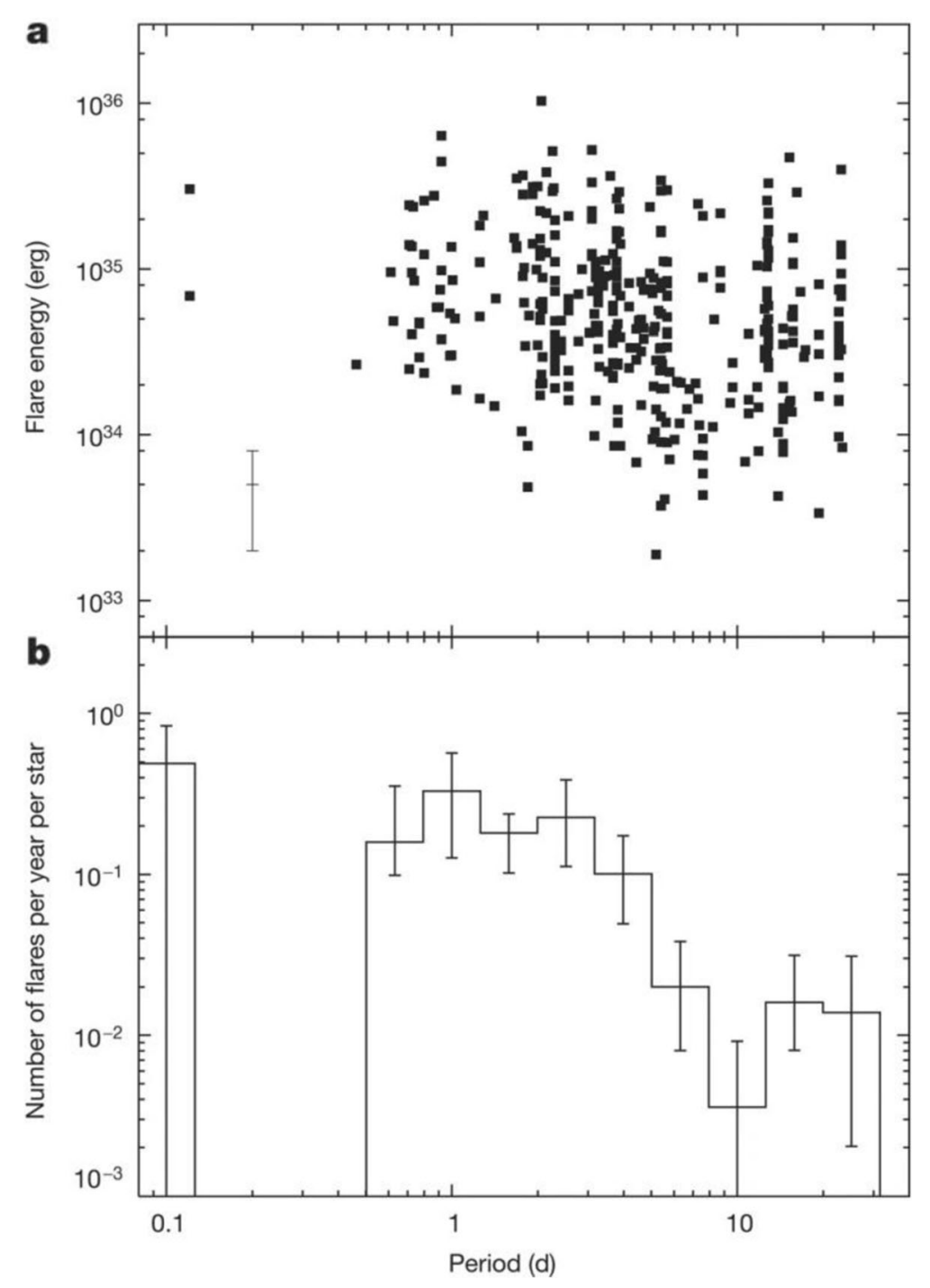

Fig. 13 Stellar superflares. The top panel shows flare energy and the bottom panel shows the frequency of occurrence of flares per year per star versus the period of brightness variation (a proxy for stellar rotation period). These observations show that extremely strong superflares occur more frequently in faster rotating stars and that solar-like stars can in principle produce extremely strong, superflares. Image reproduced from Maehara et al. (2012)

their mother star grows older and end up like Mercury or Venus. Meanwhile, planets further out will spend the first couple of billion years of their existence in deepfreeze, not advantageous for the development of life, and once they melt may not have enough time for the development of multi-cellular organisms-that took four billion years on Earth.

However, recent observations point towards the Sun, and more generally the stars that host planetary systems, for a resolution of the paradox. Most important are the results confirmed by the Mars Rovers that show that Mars has had periods with a seeming abundance of liquid water over its first billions of years (e.g., Di Achille and Hynek 2010). If both Mars and Earth both have had liquid water over their history, Occam's razor suggests we look for a common cause, i.e., a more luminous Sun than stellar evolution simulations indicate. One possibility (Willson et al. 1987; Graedel et al. 1991) for a brighter young Sun would be if the Sun had only about 5\% more mass at its origin than it has now, and consequently, has lost the excess mass through mass loss much larger than its present value of $3 \times 10^{-14} \mathrm{M}_{\text {sun }}$ /year. This hypothesis is attractive because it solves the FYSP for Earth and Mars jointly and increases the likelihood of more permanent habitable zones around other Sun-like stars, assuming that the mass loss evolution of the Sun is not exceptional. 
To promote this hypothesis to a credible theory, it has to pass a large number of tests, to see if the scenario can be made to agree with the laws of physics and whether it agrees with observations. Finally, any theory needs to make predictions about observational tests that have not been carried out yet, for unbiased verification. I will describe the first two types of tests here in that order and leave the final one for future research.

\subsection{Physical constraints}

The key question to be addressed is whether we can come up with a stellar evolution scenario that produces enough solar luminosity to keep Mars "wet" during the first about two billion years of its evolution, and that keeps Earth warm enough for liquid water over its entire existence. Minton and Malhotra (2007) have calculated what initial solar mass and subsequent mass loss is required to maintain solar luminosity more or less at the present value from the time the Sun arrived at the ZAMS. The result is a mass loss of the order of $10^{-11}$ $\mathrm{M}_{\text {sun }} /$ year for roughly the first 2.5 billion years and then rapidly declining to its current value that is a factor 300 less.

Simulations by Suzuki et al. (2013) have demonstrated that when sufficient Poynting flux enters the stellar chromosphere and corona, mass loss rates dramatically increase until they plateau at a value of about $10^{-11}$ $\mathrm{M}_{\text {Sun }} /$ year, similar to what is observed, and to the estimate by Martens (2017) based upon rotation slow-down considerations. Cranmer and Saar (2011) develop a physics-based model that is remarkably successful in reproducing the observed mass-loss rates of late-type stars over their evolution. In particular, the evolution curve for a one solar mass star produces a mass loss rate that is substantially higher than that in standard models, although still lower than that required in Minton and Malhotra (2007). However, the predicted solar luminosity curve is nearly flat at the current value going back in time, providing a possible resolution of the Faint Young Sun Paradox-which they forget to mention. We point out that there are still quite a few physical assumptions in their model that are either not yet verified, such as magnetic filling factors, or probably incorrect, e.g., that the final wind velocity equals the escape velocity. Also they neglect to address the magnetic breaking that is required for the slow-down in stellar rotation. Nevertheless, their model represents a significant contribution to the resolution of the Faint Young Sun Paradox.

Stellar evolution theory simultaneously has to account for the rapid spin-down of the Sun and Sun-like stars, from a rotation period of 4 to 5 days at the ZAMS to about a 26-day period currently. Martens (2017) pointed out that the problems of mass loss and spin-down are intimately intertwined: the torque applied to the star by its stellar wind determines the magnitude of the spindown. Simulations (Keppens and Goedbloed 2000) have shown that that torque is equal to the stellar mass loss rate times the lever arm, which is of the order of the Alfvén radius, i.e., the radius where the wind outflow velocity equals the Alfvén speed. Martens demonstrates that in order for the Sun (and Sun-like stars) to spin down from their ZAMS rotation periods, with reasonable values of the Alfvén radius of 10 to 20 solar radii, a mass loss of the order of $10^{-11} \mathrm{M}_{\text {sun }} /$ year is needed. For the Sun to slow down with its current mass loss rate, the Alfvén radius would have to be located beyond the orbit of Venus, which seems unphysical. Modern stellar evolution codes, such as MESA (Paxton et al. 2011), can include the effects of mass loss and spin down and hence various evolutionary scenarios can be simulated for comparison with observational constraints. Such simulations also produce models of the stellar interior that can deliver detailed predictions for helioseismical observables.

\subsection{Observational constraints}

The key question here is what observations can be made to verify or discard the existence of a massive solar wind through much of the Sun's history. Below we list the observations we know about that are relevant to this question:

1. The current solar mass loss rate is $3 \times 10^{-14} \mathrm{M}_{\text {sun }} /$ year.

2. The current value of the Afven radius is about 12 solar radii (Velli et al. 2016).

3. Sun-like stars at very young ages have a mass loss of $\sim 10^{-11} \mathrm{M}_{\text {sun }} /$ year. For example Gaidos et al. (2000) find, from deep observations at radio (3.6 $\mathrm{cm}$ ) wavelengths, upper limits for the mass loss rate above that value for a number of young nearby solar-type stars. Wood et al. (2005) find mass loss rates of that order for a number of young solar-type stars from the observed astrospheric absorption in Lyman-alpha.

4. Sun-like stars near the ZAMS have a rotation period of a few days at most (Barnes 2009).

5. The Sun and same aged Sun-like stars have a rotation period of 25-30 days (Barnes 2009).

6. Analysis of moon rocks that have been exposed to the solar wind for as long as they have been in existence at the lunar surface, indicate a solar mass loss about an order of magnitude higher than the current value over the last two or three billion years (Geiss and Bochsler 1991).

7. According to Sackmann and Boothroyd (2003): "Older, solar-flare irradiated grains from meteorites imply an early solar flare activity about 103 times that of the present Sun (Caffee et al. 1987); the 
associated solar wind may have been enhanced by a similar factor."

8. There is a surprising dearth of observations of mass loss rates for Sun-like stars between one and three billions years of age. There are indications of massive stellar winds sustained for $~ 3$ Gyr (Melendez et al. 2017) for Sun-like stars.

9. Helioseismic observations measure the position of the base of the Sun's convective envelope and the surface helium abundance, which slightly differ between a Sun that starts out at $1.05 \mathrm{M}_{\text {sun }}$ and constant luminosity, and one that starts out at one solar mass and an initial luminosity of $0.7 \mathrm{~L}_{\text {sun }}$, increasing linearly with time. One obvious effect is that more helium is produced in the core in the former case, which leaves fingerprints throughout the Sun (Sackmann and Boothroyd 2003). Older models for helioseismic observables for solar evolution with mass loss rates of the order of $10^{-11}$ $\mathrm{M}_{\text {sun }}$ /year produce slightly better agreement with actual helioseismic observations than those with no or current mass loss rates (Sackmann and Boothroyd 2003). However, the difference is within the error margins of those models, so no conclusion can be drawn. We have found no recent updates to these results.

More observational constraints on the mass loss history of the Sun and Sun-like stars may exist. In general, one has to be vigilant to distinguish pure observational constraints from those derived from models. For example, Cranmer (2017) concludes that "the cumulative CME mass lost by the young Sun may have been as much as $1 \%$ of a solar mass," which is relevant in this context. However, this result is based on a correlation between current CME mass loss and magnetic filling factor on the stellar surface, and hence is implied. This does not diminish this work by Cranmer, but one has to be careful in considering this an observational constraint.

We conclude that progress may be expected over the next decade in solving the FYSP: helioseismic models will be improved, fresh moon rocks will be retrieved, as well as samples for asteroids, more observations of the parameters of Sun-like stars will become available, and stellar evolution simulations with mass loss will be further explored.

\section{Conclusions}

Inspired by the VarSITI Solar Evolution Program of SCOSTEP, this review summarizes the state of the field and current understanding of solar magnetic activity fluctuations, solar extreme events, solar activity evolution, and their impacts on our space environment and planet Earth. In their individual scope, each of these topics is vast, with a diversity of literature. This review cannot do justice to these individual sub-topics but has rather focused on the interconnected themes and progress therein.

It is our hope that this will serve as a useful source of ideas, information, and references which would instigate future investigations of solar-stellar activity and attempts to understand their impact on planets and exoplanets.

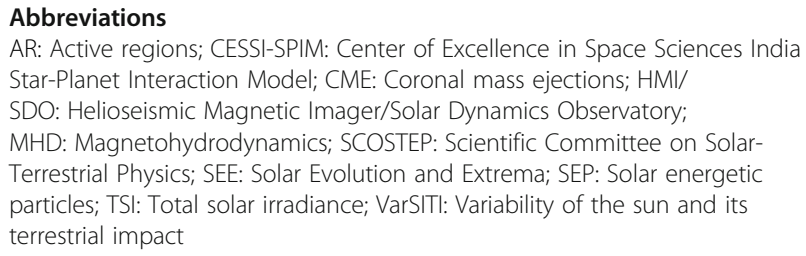

\section{Acknowledgements}

We acknowledge scientific discussions facilitated by the SCOSTEP VarSITI program and the International Astronomical Union (IAU) Working Group on Impact of Magnetic Activity on Solar and Stellar Environments. We are thankful to llya Usoskin, Aline Vidotto, and Kazunari Shibata for providing Fig. 6, Fig. 12, and Fig. 13, respectively. CESSI is funded by the Ministry of Education of the Government of India under the Frontier Areas of Science and Technology (FAST) scheme.

\section{Authors' contributions}

D.N. led the writing of this review with contributions from P.M. Some of the figures were generated by S.D. Planning of the contents of this review were done by D.N., P.M., V.O., and K.G. All authors have read and approved the final manuscript.

\section{Authors' information}

D.N., P.M., and V.O. co-lead the Solar Evolution and Extrema program of SCOSTEP VarSITI.

\section{Funding}

The Center of Excellence in Space Sciences India (CESSI) is funded by the Ministry of Education of the Government of India.

\section{Availability of data and materials}

Data which feature in plots highlighted in this manuscript are available from the sources cited at the relevant sections. Furthermore, specific data sources used for some of the plots are available through the following online data repositories.

Sunspot Time Series Data:

http://www.sidc.be/silso/datafiles

Total Solar Irradiance Data:

ftp://ftp.pmodwrc.ch/pub/data/irradiance/composite/DataPlots/

composite_42_65_1709.dat

Cosmic Ray Data:

http://cidas.isee.nagoya-u.ac.jp/WDCCR/

\section{Declarations}

\section{Competing interests}

The authors declare that they have no competing interests.

\section{Author details}

${ }^{1}$ Center of Excellence in Space Sciences India, Indian Institute of Science Education and Research Kolkata, Mohanpur, West Bengal 741246, India. ${ }^{2}$ Department of Physical Sciences, Indian Institute of Science Education and Research Kolkata, Mohanpur, West Bengal 741246, India. ${ }^{3}$ Department of Physics \& Astronomy, Georgia State University, 25 Park Place, 6th Floor, Atlanta, GA 30303, USA. ${ }^{4}$ Pushkov Institute of Solar-Terrestrial Physics, lonosphere, and Radio Wave Propagation, Kaluzhskoe shosse 4, Troitsk, 
Moscow 108840, Russia. ${ }^{5}$ Space Research and Technology Institute, Bulgarian Academy of Sciences, Sofia, Bulgaria.

\section{Received: 14 January 2021 Accepted: 20 May 2021}

Published online: 05 July 2021

\section{References}

Achille DG, Hynek BM (2010) Ancient ocean on Mars supported by global distribution of deltas and valleys. Nat Geosci 3:459-463

Ahuir J, Brun AS, Strugarek A (2020) From stellar coronae to gyrochronology: theoretical and observational exploration. Astronomy Astrophysics 635:A170

Alvarado-Gómez JD, Drake JJ, Garraffo C, Cohen O, Poppenhaeger K, Yadav RK, Moschou SP (2020) An Earth-like stellar wind environment for Proxima Centauri. Astrophys J Lett 902(1):L9. https://doi.org/10.3847/2041-8213/a bb885

Aulanier G, Démoulin P, Schrijver CJ, Janvier M, Paria E, Schmieder B (2013) The standard flare model in three dimensions. II. Upper limit on solar fare energy. Astrophysical J 549:A66

Babcock HW (1961) The topology of the Sun's magnetic field and the 22-year cycle. Astrophysical J 133:572. https://doi.org/10.1086/147060

Barnes SA (2009) Gyrochronology and its usage for main sequence field star ages. IAU Symp 258:345-356

Basak A, Nandy D (2021) Star planet interaction simulations of the imposed magnetospheres of Mars and Mars-like exoplanets. Monthly Notices R Astronomical Soc 502:3569-3581

Beer J, Tobias SM, Weiss NO (2017) On long-term modulation of the Sun's magnetic cycle. Monthly Notices Royal Astronomical Soc 473:1596-1602

Benz, AO (2017) Flare Observations. Living Reviews in Solar Physics 14:2.

Bhowmik P, Nandy D (2018) Prediction of the strength and timing of sunspot cycle 25 reveal decadal-scale space environmental conditions. Nat Commun 9(1):5209. https://doi.org/10.1038/s41467-018-07690-0

Brun AS, Garcia RA, Houdek G, Nandy D, Pinsonneault M (2015) The solar-stellar connection. Space Sci Rev 196:303-356

Brun AS, Miesch MS, Toomre J (2004) Global-scale turbulent convection and magnetic dynamo action in the solar envelope. Astrophysical J 614(2):10731098. https://doi.org/10.1086/423835

Caffee M, Hohenberg C, Swindle T (1987) Evidence in meteorites for an active early Sun. Astrophysical J 313:L31. https://doi.org/10.1086/184826

Cameron R, Schüssler M (2015) The crucial role of surface magnetic fields for the solar dynamo. Science 347(6228):1333-1335. https://doi.org/10.1126/ science. 1261470

Cameron RH, Jiang J, Schüssler M (2016) Solar cycle 25: another moderate cycle? Astrophysical J Lett 823(2):L22. https://doi.org/10.3847/2041-8205/823/2/L22

Carolan S, Vidotto AA, Loesch C, Coogan P (2019) The evolution of Earth's magnetosphere during the solar main sequence. Monthly Notices $R$ Astronomical Soc 489:5784-5801, 4. https://doi.org/10.1093/mnras/stz2422

Charbonneau P (2020) Dynamo models of the solar cycle. Living Rev Solar Phys 17(1):4. https://doi.org/10.1007/s41116-020-00025-6

Chatterjee P, Nandy D, Choudhuri AR (2004) Full-sphere simulations of a circulation-dominated solar dynamo: exploring the parity issue. Astronomy Astrophysics 427(3):1019-1030. https://doi.org/10.1051/0004-6361:20041199

Choudhuri AR, Chatterjee P, Jiang J (2007) Predicting solar cycle 24 with a solar dynamo model. Physi Rev Lett 98(13):131103. https://doi.org/10.1103/ PhysRevLett.98.131103

Choudhuri AR, Schüssler M, Dikpati M (1995) The solar dynamo with meridional circulation. Astronomy Astrophysics 303:L29-L32

Cohen J, Screen JA, Furtado JC, Barlow M, Whittleston D, Coumou D, Francis J, Dethloff K, Entekhabi D, Overland J, Jones J (2014) Recent Arctic amplification and extreme mid-latitude weather. Nature Geosci 7(9):627-637. https://doi.org/10.1038/ngeo2234

Cranmer S (2017) Mass-loss rates from coronal mass ejections: a predictive theoretical model for solar-type stars. Astrophysical J 840:114-124

Cranmer SR, Saar SH (2011) Testing a predictive theoretical model for the massloss rates of cool stars. Astrophys. J. 741(1):54-76. https://doi.org/10.1088/ 0004-637X/741/1/54

Das SB, Basak A, Nandy D, Vaidya B (2019) Modeling Star-Planet Interactions in far-out planetary and exoplanetary systems. Astrophysical J 877(2):80. https:// doi.org/10.3847/1538-4357/ab18ad

Dasi-Espuig M, Solanki SK, Krivova NA, Cameron R, Peñuela T (2010) Sunspot group tilt angles and the strength of the solar cycle. Astronomy Astrophysics 518:A7. https://doi.org/10.1051/0004-6361/201014301
De Jager C (2005) Solar Forcing of Climate. 1: Solar Variability, vol 120, pp 197-241

Deeg HJ, Belmonte JA (2018) Handbook of Exoplanets. Springer, Switzerland. https://doi.org/10.1007/978-3-319-55333-7

DeRosa M, Brun AS, Hoeksema JT (2012) Solar magnetic field reversals and the role of dynamo families. Astrophysical J 757(96):1. https://doi.org/10.1088/ 0004-637X/757/1/96

Dikpati M, Charbonneau P (1999) A Babcock-Leighton flux transport dynamo with solar-like differential rotation. Astrophysical J 518(1):508-520. https://doi. org/10.1086/307269

Dikpati M, de Toma G, Gilman PA (2006) Predicting the strength of solar cycle 24 using a flux-transport dynamo-based tool. Geophys Res Lett 33:5102

Durney BR (1995) On a Babcock-Leighton dynamo model with a deep-seated generating layer for the toroidal magnetic field. Solar Phys 160(2):213-235. https://doi.org/10.1007/BF00732805

Eddy JA (1976) The Maunder Minimum. Science 192(4245):1189-1202. https://doi. org/10.1126/science.192.4245.1189

Feulner G (2012) The faint young Sun problem. Revi Geophys 50:RG2006

Fionnagáin Ó, Vidotto D, Petit AA, Folsom P, Jeffers CP, Marsden SV, Morin SC, do Nascimento J, JD, BCool Collaboration (2019) Monthly Notices of the Royal Astronomical Society. Volume 483:873-886

Fionnagáin ÓD, Vidotto AA (2018) The solar wind in time: a change in the behavior of older winds? Monthly Notices R Astronomical Soc 476(2): 2465-2475. https://doi.org/10.1093/mnras/sty394

Fröhlich C (2006) Solar irradiance variability since 1978-revision of the PMOD composite during solar cycle 21. Space Sci Rev 125:53-65

Gaidos EJ, Güdel M, Blake GA (2000) The Faint Young Sun Paradox: an observational test of an alternative solar model. Geophys Res Lett 27(4):501503. https://doi.org/10.1029/1999GL010740

Gallet F, Charbonnel C, Amard L, Brun S, Palacios A, Mathis S (2017) Impacts of stellar evolution and dynamics on the habitable zone: the role of rotation and magnetic activity. Astronomy Astrophysics 597:A14. https://doi.org/10.1 051/0004-6361/201629034

Geiss, J, and Bochsler, P (1991), in the Sun in time, ed. Sonnett CP, Giampapa MS, Matthews MS. Tucson: Univ. Arizona Press 98

Ghizaru M, Charbonneau P, Smolarkiewicz PK (2010) Magnetic cycles in global large-eddy simulations of solar convection. Astrophysical J Lett 715(2):L133L137. https://doi.org/10.1088/2041-8205/715/2/L133

Gopalswamy N (2016) History and development of coronal mass ejections as a key player in solar terrestrial relationship. Geosci Lett 3(1):8-26. https://doi. org/10.1186/s40562-016-0039-2

Gopalswamy N (2017) Solar origins and statistics of extremes. In: Buzulukova N (ed) Extreme events in geospace. Origins, predictability, and consequences. Elsevier, Amsterdam

Graedel TE, Sackmann IJ, Boothroyd AI (1991) Early solar mass loss: a potential solution to the weak sun paradox. Geophysical Res Lett 18(10):1881-1884. https://doi.org/10.1029/91GL02314

Gronoff G, Arras P, Baraka S, Bell JM, Cessateur G, Cohen O, Curry SM, Drake JJ, Elrod M, Erwin J, Garcia-Sage K, Garraffo C, Glocer A, Heavens NG, Lovato K, Maggiolo R, Parkinson CD, Simon Wedlund C, Weimer DR, Moore WB (2020) Atmospheric escape processes and planetary atmospheric evolution. J Geophysical Res 125:e2019JA027639

Guerrero G, Gouveia d, Dal Pino EM (2008) Turbulent magnetic pumping in a Babcock-Leighton solar dynamo model. Astronomy Astrophysics 485(1):267273. https://doi.org/10.1051/0004-6361:200809351

Guerrero G, Smolarkiewicz PK, Kosovichev AG, Mansour NN (2013) Differential rotation in solar-like stars from global simulations. Astrophysical J 779(2):176. https://doi.org/10.1088/0004-637X/779/2/176

Guerrero G, Zaire B, Smolarkiewicz PK, de Gouveia Dal Pino EM, Kosovichev AG, Mansour NN (2019) What sets the magnetic field strength and cycle period in solar-type stars? Astrophysical J 880(1):6. https://doi.org/10.3847/1538-4357/ab224a

Guerrero GA, Muñoz JD (2004) Kinematic solar dynamo models with a deep meridional flow. Monthly Notices R Astronomical Soc 350(1):317-322. https:// doi.org/10.1111/j.1365-2966.2004.07655.x

Guinan, EF, and Engle, SG (2007) Evolution over time of magnetic dynamo driven UV \& X-ray emissions of dG-M stars and effects on hosted planets. Oral Contribution to IAU 26th General Assembly Joint Discussion 4 - The Ultraviolet Universe: Stars from Birth to Death (arXiv: 0711.1530)

Haigh JD (2007) The Sun and the Earth's climate. Living Rev Solar Phys 4:2

Hale GE, Ellerman F, Nicholson SB, Joy AH (1919) The magnetic polarity of sunspots. Astrophysical J 49:153. https://doi.org/10.1086/142452 
Hathaway D (2015) The Solar Cycle. Living Rev Solar Phys 7:1

Hazra S, Brun AS, Nandy D (2020) Does the mean-field a effect have any impact on the memory of the solar cycle? Astronomy Astrophysics 642:A51. https:// doi.org/10.1051/0004-6361/201937287

Hazra S, Nandy D (2016) A proposed paradigm for solar cycle dynamics mediated via turbulent pumping of magnetic flux in Babcock-Leighton-type solar dynamos. Astrophysical J 832(1):9. https://doi.org/10.3847/0004-637X/ $832 / 1 / 9$

Hazra S, Nandy D (2019) The origin of parity changes in the solar cycle. Monthly Notices R Astronomical Soc 489(3):4329-4337. https://doi.org/10.1093/mnras/ stz2476

Hazra S, Passos D, Nandy D (2014) A stochastically forced time delay solar dynamo model: self-consistent recovery from a Maunder-like grand minimum necessitates a mean-field alpha effect. Astrophysical J 789(1):5. https://doi.org/10.1088/0004-637X/789/1/5

Hotta H, Rempel M, Yokoyama T (2016) Large-scale magnetic fields at high Reynolds numbers in magnetohydrodynamic simulations. Science 351(6280): 1427-1430. https://doi.org/10.1126/science.aad1893

lijima H, Hotta H, Imada S, Kusano K, Shiota D (2017) Improvement of solar-cycle prediction: plateau of solar axial dipole moment. Astronomy Astrophysics 607:L2

Intergovernmental Panel on Climate Change Assessment Report (2013) Climate Change 2013: The Physical Science Basis. Switzerland, IPCC https://www.ipcc. ch/report/ar5/wg1/

Jiang J, Wang JX, Jiao QR, Cao JB (2018) Predictability of the solar cycle over one cycle. Astrophysical J 863(159):2. https://doi.org/10.3847/1538-4357/aad197

Jouve L, Proctor MRE, Lesur G (2010) Buoyancy-induced time delays in BabcockLeighton flux-transport dynamo models. Astronomy Astrophysics 519:A68. https://doi.org/10.1051/0004-6361/201014455

Käpylä MJ, Käpylä PJ, Olspert N, Brandenburg A, Warnecke J, Karak BB, Pelt J (2016) Multiple dynamo modes as a mechanism for long-term solar activity variations. Astronomy Astrophysics 589:A56. https://doi.org/10.1051/0004-63 61/201527002

Käpylä PJ, Käpylä MJ, Olspert N, Warnecke J, Brandenburg A (2017) Convectiondriven spherical shell dynamos at varying Prandtl numbers. Astronomy Astrophysics 599:A4. https://doi.org/10.1051/0004-6361/201628973

Käpylä PJ, Korpi MJ, Ossendrijver M, Stix M (2006a) Magnetoconvection and dynamo coefficients III. a-effect and magnetic pumping in the rapid rotation regime. Astronomy and Astrophysics 455(2):401-412. https://doi.org/10.1051/ 0004-6361:20064972

Käpylä PJ, Korpi MJ, Tuominen I (2006b) Solar dynamo models with a-effect and turbulent pumping from local $3 \mathrm{~d}$ convection calculations. Astron Nachr 327(9):884. https://doi.org/10.1002/asna.200610636

Karak BB, Miesch M (2017) Solar cycle variability induced by tilt angle scatter in a Babcock-Leighton solar dynamo model. Astrophysical J 847(69):1. https://doi. org/10.3847/1538-4357/aa8636

Karak BB, Nandy D (2012) Turbulent pumping of magnetic flux reduces solar cycle memory and thus impacts predictability of the Sun's activity. Astrophysical J Lett 761(1):L13. https://doi.org/10.1088/2041-8205/761/1/L13

Kasting JF (1989) Long-term stability of the earth's climate. Glob Planet Change 1(1-2):83-95. https://doi.org/10.1016/0921-8181(89)90017-9

Kasting JF (2004) Evolution of Earth's Atmosphere and Climate. AGU Fall Meeting Abstracts

Kasting JF, Toon OB, Pollack JB (1988) How climate evolved on the terrestrial planets. Sci Am 258:46-53

Keppens R, Goedbloed JP (2000) Stellar winds, dead zones, and coronal mass ejections. Astrophysical J 530:1036-1048

Krivova NA, Balmaceda L, Solanki SK (2007) Reconstruction of solar total irradiance since 1700 from the surface magnetic flux. Astronomy Astrophysics 467(1):335-346. https://doi.org/10.1051/0004-6361:20066725

Kumar R, Jouve L, Nandy D (2019) A 3D kinematic Babcock-Leighton solar dynamo model sustained by dynamic magnetic buoyancy and flux transport processes. Astronomy Astrophysics 623:A54. https://doi.org/10.1051/0004-6361/201834705

Labonville F, Charbonneau P, Lemerle A (2019) A dynamo-based forecast of solar cycle 25. Solar Physics 294(6):82. https://doi.org/10.1007/s11207-019-1480-0

Lammer H, Güdel M, Kulikov Y, Ribas I, Zaqarashvili TV, Khodachenko ML, Kislyakova KG, Gröller H, Odert P, Leitzinger M, Fichtinger B, Krauss S, Hausleitner W, Holmström M, Sanz-Forcada J, Lichtenegger HIM, Hanslmeier A, Shematovich VI, Bisikalo D, Rauer H, Fridlund M (2012) Variability of solar/ stellar activity and magnetic field and its influence on planetary atmosphere evolution. Earth Planet Space 64:13
Leighton RB (1969) A magneto-kinematic model of the solar cycle. Astrophysical J 156:1. https://doi.org/10.1086/149943

Lekshmi B, Nandy D, Antia HM (2018) Asymmetry in solar torsional oscillation and the sunspot cycle. Astrophysical J 861:121

Lekshmi B, Nandy D, Antia HM (2019) Hemispheric asymmetry in meridional flow and the sunspot cycle. Monthly Notices R Astronomical Soc 489(1):714. https://doi.org/10.1093/mnras/stz2168

Lockwood M, Owens MJ, Barnard L (2014) Centennial variations in sunspot number, open solar flux, and streamer belt width: 1. Correction of the sunspot number record since 1874. J Geophys Res Space Phys 119(7):51725182. https://doi.org/10.1002/2014JA019970

Maehara H, Shibayama T, Notsu S, Notsu Y, Nagao T, Kusaba S, Honda S, Nogami D, Shibata K (2012) Superflares on solar-type stars. Nature 485(7399):478-448. https://doi.org/10.1038/nature11063

Martens PCH (2017) The Faint Young Sun and Faint Young Stars Paradox. IAU Symp 328:350-355

McCracken KG (2007) Heliomagnetic field near Earth, 1428-2005. J Geophys Res 112:A09106

Melendez J, dos Santos LA, Freitas FC (2017) Improved rotation-activity-age relations in Sun-like stars. IAU Symp 328:274-281

Metcalfe TS, van Saders J (2020) Comment on "The Sun is less active than other solar-like stars". arXiv:2007.04416

Mininni PD, Gómez DO, Mindlin GB (2002) Biorthogonal decomposition techniques unveil the nature of the irregularities observed in the solar cycle. Phys Rev Lett 89(6):061101. https://doi.org/10.1103/PhysRevLett.89.061101

Minton DA, Malhotra R (2007) Assessing the Massive Young Sun Hypothesis to solve the Warm Young Earth Puzzle. Astrophysical J 660(2):1700-1706. https://doi.org/10.1086/514331

Mishra RK, Marhas KK (2019) Meteoritic evidence of a late superflare as source of 7Be in the early Solar System. Nat Astronomy 3:498-505

Miyahara H, Kitazawa K, Nagaya K, Yokoyama Y, Matsuzaki H, Masuda K, Nakamura T, Muraki Y (2010) Is the Sun heading for another Maunder minimum? Precursors of the grand solar minima. J Cosmol 8:1970-1982

Miyahara H, Masuda K, Muraki Y, Furuzawa H, Menjo H, Nakamura T (2004) Cyclicity of solar activity during the Maunder minimum deduced from radiocarbon content. Solar Physics 224(1-2):317-322. https://doi.org/10.1007/ s11207-005-6501-5

Muñoz-Jaramillo A, Dasi-Espuig M, Balmaceda LA, DeLuca EE (2013) Solar cycle propagation, memory, and prediction: insights from a century of magnetic proxies. Astrophysical J Lett 767(2):L25. https://doi.org/10.1088/2041-8205/ 767/2/L25

Muñoz-Jaramillo A, Nandy D, Martens PCH (2009) Helioseismic data inclusion in solar dynamo models. Astrophysical J 698(1):461-478. https://doi.org/10.1 088/0004-637X/698/1/461

Muñoz-Jaramillo A, Nandy D, Martens PCH (2011) Magnetic quenching of turbulent diffusivity: reconciling mixing-length theory estimates with kinematic dynamo models of the solar cycle. Astrophysical J Lett 727(1):L23. https://doi.org/10.1088/2041-8205/727/1/L23

Muñoz-Jaramillo A, Nandy D, Martens PCH, Yeates AR (2010) A double-ring algorithm for modeling solar active regions: unifying kinematic dynamo models and surface flux-transport simulations. Astrophysical J Lett 720(1): L20-L25. https://doi.org/10.1088/2041-8205/720/1/L20

Mursula K, Usoskin IG, Kovaltsov GA (2001) Persistent 22-year cycle in sunspot activity: evidence for a relic solar magnetic field. Solar Phys 198(1):51-56. https://doi.org/10.1023/A:1005218414790

Mursula K, Usoskin IG, Maris G (2007) Introduction to space climate. Adv Space Res 40(7):885-887. https://doi.org/10.1016/j.asr.2007.07.046

Nagy M, Lemerle A, Labonville F, Petrovay K, Charbonneau P (2017) The effect of "rogue" active regions on the solar cycle. Solar Phys 292(11):167. https://doi. org/10.1007/s11207-017-1194-0

Nandy D (2002) Constraints on the solar internal magnetic field from a buoyancy driven solar dynamo. Astrophys Space Sci 282(1):209-219. https://doi.org/10.1 023/A:1021632522168

Nandy D (2004) Exploring magnetic activity from the Sun to the stars. Solar Phys 224(1-2):161-169. https://doi.org/10.1007/s11207-005-4990-x

Nandy, D (2021) Progress in solar cycle predictions: sunspot cycles 24-25 in Perspective. Solar Physics, in press (arXiv:2009.01908), 296, 3, doi: 10.1007/ s11207-021-01797-2

Nandy D, Bhatnagar A, Pal S (2020) Sunspot cycle 25 is brewing: early signs herald its onset. Res Notes AAS 4(2):30. https://doi.org/10.3847/2515-5172/a b79a1 
Nandy D, Choudhuri AR (2001) Toward a mean-field formulation of the Babcock-Leighton type solar dynamo I. a-coefficient versus Durney's double-ring approach. Astrophysical Journal 551(1):576-585. https://doi. org/10.1086/320057

Nandy D, Choudhuri AR (2002) Explaining the latitudinal distribution of sunspots with deep meridional flow. Science 296(5573):1671-1673. https://doi.org/1 $0.1126 /$ science. 1070955

Nandy D, Martens PCH (2007) Space climate and the solar-stellar connection: what can we learn from the stars about long-term solar variability? Adv Space Res 40(7):891-898. https://doi.org/10.1016/j.asr.2007.01.079

Nandy D, Muñoz-Jaramillo A, Martens PCH (2011) The unusual minimum of sunspot cycle 23 caused by meridional plasma flow variations. Nature 471(7336):80-82. https://doi.org/10.1038/nature09786

Nelson NJ, Brown BP, Brun AS, Miesch MS, Toomre J (2013) Magnetic wreaths and cycles in convective dynamos. Astrophysical J 762(2):73. https://doi.org/1 0.1088/0004-637X/762/2/73

Notsu Y, Maehara H, Honda S, Hawley SL, Davenport JRA, Namekata K, Notsu S, Ikuta K, Nogami D, Shibata K (2019) Do Kepler superflare stars really include slowly rotating sun-like stars?-results using APO $3.5 \mathrm{~m}$ telescope spectroscopic observations and Gaia-DR2 data. Astrophysical J 876(1):58-97. https://doi.org/10.3847/1538-4357/ab14e6

Notsu Y, Shibayama T, Maehara H, Notsu S, Nagao T, Honda S, Ishii TT, Nogami D, Shibata K (2013) Superflares on solar-type stars observed with Kepler ii. photometric variability of superflare-generating stars: a signature of stellar rotation and starspots. Astrophysical J 771(2):127-137. https://doi.org/10.1 088/0004-637X/771/2/127

Obridko V, Georgieva K (2018) Expected evolution of solar activity in the following decades. J Atmospheric Solar-Terrestrial Phys 176:1-82. https://doi. org/10.1016/j.jastp.2018.08.001

Obridko VN, Sokoloff DD, Shelting BD, Shibalova AS, Livshits IM (2020) Cyclic variations in the main components of the solar large-scale magnetic field. Monthly Notices R Astronomical Soc 492(4):5582-5591. https://doi.org/10.1 093/mnras/staa147

Okamoto, S, Notsu, Y, Maehara, H, Namekata, K, Honda, S, Ikuta, K, Nogami, D, and Shibata, K, (2021) Statistical properties of superflares on solar-type stars: results using all of the Kepler primary mission data. Astrophysical J, in press 906, 2, doi: https://doi.org/10.3847/1538-4357/abc8f5

Owens M, Cliver E, McCracken K, Beer J, Barnard L, Lockwood M, Rouillard A, Passos D, Riley P, Usoskin I, Wang YM (2016) Near-earth heliospheric magnetic field intensity since 1750. Part 1: Sunspot and geomagnetic reconstructions. J Geophys Res 121:6048-6063

Owens MJ, Lockwood M, Riley P (2017) Global solar wind variations over the last four centuries. Sci Rep 7(1):41548. https://doi.org/10.1038/srep41548

Pal S, Dash S, Nandy D (2020) Flux erosion of magnetic clouds by reconnection with the Sun's open flux. Geophys Res Lett 47:e2019GL086372

Parker EN (1955a) Hydromagnetic dynamo models. Astrophysical J 122:293-314. https://doi.org/10.1086/146087

Parker EN (1955b) The formation of sunspots from the solar toroidal field. Astrophys J 121:491. https://doi.org/10.1086/146010

Passos D, Nandy D, Hazra S, Lopes I (2014) A solar dynamo model driven by mean-field alpha and Babcock-Leighton sources: fluctuations, grand-minimamaxima, and hemispheric asymmetry in sunspot cycles. Astronomy Astrophysics 563:A18. https://doi.org/10.1051/0004-6361/201322635

Paxton B, Bildsten L, Dotter A, Herwig F, Lesaffre P, Timmes F (2011) Modules for Experiments in Stellar Astrophysics (MESA). Astrophysical J Suppl Series 192(1):3-38. https://doi.org/10.1088/0067-0049/192/1/3

Pesnell WD (2008) Predictions of solar cycle 24. Solar Phys 252(1):209-220. https://doi.org/10.1007/s11207-008-9252-2

Petrovay K (2020) Solar cycle prediction. Living Rev Solar Phys 17(1):2. https://doi. org/10.1007/s41116-020-0022-Z

Poppenhaeger K, Lenz LF, Reiners A, Schmitt JHMM, Shkolnik E (2011) A search for star-planet interactions in the $U$ Andromedae system at X-ray and optical wavelengths. Astronomy Astrophysics 528:A58. https://doi.org/10.1051/0004$6361 / 201016008$

Pulkkinen T (2007) Space weather: the terrestrial perspective. Living Rev Solar Phys 4:1

Raynaud R, Tobias SM (2016) Convective dynamo action in a spherical shell: symmetries and modulation. J Fluid Mechanics 799:1-12

Reinhold T, Shapiro Al, Solanki SK, Montet BT, Krivova NA, Cameron RH, AmazoGómez EM (2020a) The Sun is less active than other solar-like stars. Science 368:518-521. https://doi.org/10.1126/science.aay3821
Reinhold T, Shapiro Al, Solanki SK, Montet BT, Krivova, NA, Cameron RH, AmazoGómez, EM (2020b) Reply to the comment of T. Metcalfe and J. van Saders on the Science report "The Sun is less active than other solar-like stars". arXiv: 2007.04817

Rodgers-Lee D, Vidotto AA, Taylor AM, Rimmer PB, Downes TP (2020) The Galactic cosmic ray intensity at the evolving Earth and young exoplanets. Monthly Notices R Astronomical Soc 499(2):2124-2137. https://doi.org/10.1 093/mnras/staa2737

Sackmann J, Boothroyd A (2003) Our Sun. V. A bright young Sun consistent with helioseismology and warm temperatures on ancient Earth and Mars. Astrophysical J 583:1024

Sakata R, Seki K, Sakai S, Terada N, Shinagawa H, Tanaka T (2020) Effects of an intrinsic magnetic field on ion loss from ancient mars based on multispecies MHD simulations. J Geophys Res 125:e2019JA026945

Schatten KH, Scherrer PH, Svalgaard L, Wilcox JM (1978) Using dynamo theory to predict the sunspot number during solar cycle 21. Geophys Res Lett 5(5): 411-414. https://doi.org/10.1029/GL005i005p00411

Schmieder B (2018) Extreme solar storms based on solar magnetic field. J Atmospheric Solar-Terrestrial Physics 180:46-51

Schrijver CJ, Kauristie K, Aylward AD, Denardini CM, Gibson SE, Glover A, Gopalswamy N, Grande M, Hapgood M, Heynderickx D, Jakowski N, Kalegaev W, Lapenta G, Linker JA, Liu S, Mandrini CH, Mann IR, Nagatsuma T, Nandy D, Obara T, Paul O'Brien T, Onsager T, Opgenoorth HJ, Terkildsen M, Valladares CE, Vilmer N (2015) Understanding space weather to shield society: a global road map for 2015-2025 commissioned by COSPAR and ILWS. Adv Space Res 55:2745-2807

Schwabe H (1844) Sonnen-Beobachtungen im Jahre 1843, Astron. Nachr. 21: 233-236

Schwenn R (2006) Space weather: the solar perspective. Living Rev Solar Phys 3:2

Shapiro AV, Shapiro Al, Gizon L, Krivova NA, Solanki SK (2020) Solar-cycle irradiance variations over the last four billion years. Astronomy Astrophysics 636:A83. https://doi.org/10.1051/0004-6361/201937128

Shibayama T, Maehara H, Notsu S, Notsu Y, Nagao T, Honda S, Ishii TT, Nogami D, Shibata K (2013) Superflares on solar-type stars observed with Kepler. I. Statistical properties of superflares. Astrophysical J Suppl Series 209:1

Strugarek A (2016) Assessing magnetic torques and energy fluxes in close-in star-planet systems. Astrophysical J 833(140):2. https://doi.org/10.3847/153 8-4357/833/2/140

Strugarek A, Brun AS, Donati JF, Moutou C, Réville V (2019) Chasing Star-Planet Magnetic Interactions: The Case of Kepler-78. Astrophysical J 881, 136(2). https://doi.org/10.3847/1538-4357/ab2ed5

Strugarek A, Brun AS, Matt SP, Réville V (2014) On the diversity of magnetic interactions in close-in star-planet systems. Astrophysical J 795(86):1. https:// doi.org/10.1088/0004-637X/795/1/86

Suzuki T, Imada S, Kataoka R, Kato Y, Matsumoto T, Miyahara H, Tsuneta S (2013) Saturation of stellar winds from young suns. Publ Astron Soc Japan 65(5):9821. https://doi.org/10.1093/pasj/65.5.98

Temmer M, Veronig A, Hanslmeier A (2002) Hemispheric sunspot numbers Rn and Rs: catalogue and N-S asymmetry analysis. Astronomy Astrophysics 390(2):707-715. https://doi.org/10.1051/0004-6361:20020758

Tobias SM (2002) Modulation of solar and stellar dynamos. Astronomische Nachrichten 323(3-4):417-423. https://doi.org/10.1002/1521-3994(2002 08)323:3/4<417::AID-ASNA417>3.0.CO;2-U

Tripathi, B, Nandy, D, and Banerjee, S (2021) Stellar mid-life crisis: subcritical magnetic dynamos of solar-like stars and the break-down of gyrochronology. Monthly Notices R Astronomical Soc Lett. in press

Upton LA, Hathaway DH (2018) An updated solar cycle 25 prediction with AFT: the modern minimum. Geophysical Res Lett 45(16):8091-8095. https://doi. org/10.1029/2018GL078387

Usoskin IG (2017) A history of solar activity over millennia. Living Rev Solar Phys 14(1):3. https://doi.org/10.1007/s41116-017-0006-9

Usoskin IG, Arlt R, Asvestari E, Hawkins E, Käpylä M, Kovaltsov GA, Krivova N, Lockwood M, Mursula K, O'Reilly J, Owens M, Scott C, Sokoloff DD, Solanki SK, Soon W, Vaquero JM (2015) The Maunder minimum (1645-1715) was indeed a grand minimum: a reassessment of multiple datasets. Astronomy Astrophysics A95:581-600

Usoskin IG, Hulot G, Gallet Y, Roth R, Licht A, Joos F, Kovaltsov GA, Thebault E, Khokhlov A (2014) Evidence for distinct modes of solar activity. Astronomy Astrophysics 562:L10. https://doi.org/10.1051/0004-6361/201423391

Usoskin IG, Solanki SK, Kovaltsov GA (2007) Grand minima and maxima of solar activity: new observational constraints. Astronomy Astrophysics 471(1):301309. https://doi.org/10.1051/0004-6361:20077704 
van Saders JL, Ceillier T, Metcalfe TS, Silva Aguirre V, Pinsonneault MH, García RA, Mathur S, Davies GR (2016) Weakened magnetic braking as the origin of anomalously rapid rotation in old field stars. Nature 529(7585):181-184. https://doi.org/10.1038/nature16168

Velli M, Tenerani A, DeForest C (2016) Inward motions in the outer solar corona between 6 And 12 R: evidence for waves or magnetic reconnection jets? AAS/Solar Phys Div Meet 47:402.05

Versteegh GJM (2005) Solar Forcing of Climate. 2: Evidence from the Past. Space Sci Rev 120(3-4):243-286. https://doi.org/10.1007/s11214-005-7047-4

Vidotto AA, Gregory SG, Jardine M, Donati JF, Petit P, Morin J, Folsom CP, Bouvier J, Cameron AC, Hussain G, Marsden S, Waite IA, Fares R, Jeffers S, do Nascimento JD Jr (2014) Stellar magnetism: empirical trends with age and rotation. Monthly Notices R Astronomical Soc 441(3):2361-2374. https://doi. org/10.1093/mnras/stu728

Vieira LEA, Solanki SK, Krivova NA, Usoskin IG (2011) Evolution of the solar irradiance during the Holocene. Astronomy Astrophysics 531:A6. https://doi. org/10.1051/0004-6361/201015843

Weiss NO, Tobias SM (2016) Supermodulation of the Sun's magnetic activity: the effects of symmetry changes. Monthly Notices R Astronomical Soc 456(3): 2654-2661. https://doi.org/10.1093/mnras/stv2769

Willson LA, Bowen GH, Struck-Marcel C (1987) Mass loss on the main sequence. Comments on Modern Phys 12:17-34

Wilmot-Smith AL, Martens PCH, Nandy D, Priest ER, Tobias SM (2005) Low-order stellar dynamo models. Monthly Notices R Astronomical Soc 363(4):11671172. https://doi.org/10.1111/j.1365-2966.2005.09514.x

Wilmot-Smith AL, Nandy D, Hornig G, Martens PCH (2006) A time delay model for solar and stellar dynamos. Astrophysical J 652(1):696-708. https://doi. org/10.1086/508013

Wolk SJ, Harnden FR Jr, Flaccomio E, Micela G, Favata F, Shang H, Feigelson (eds) (2005) Stellar activity on the young suns of Orion: COUP Observations of K57 Pre-Main-Sequence Stars. Astrophysical J 160(2):423-449. https://doi.org/1 $0.1086 / 432099$

Wood BE, Müller H-R, Zank GP, Linsky JL, Redfield S (2005) New mass-loss measurements from astrospheric Lyman-alpha absorption. Astrophys J Lett 628(2):L143-L146. https://doi.org/10.1086/432716

Wu CJ, Krivova NA, Solanki SK, Usoskin IG (2018) Solar total and spectral irradiance reconstruction over the last 9000 years. Astronomy Astrophysics 620:A12

Yeates AR, Muñoz-Jaramillo A (2013) Kinematic active region formation in a three-dimensional solar dynamo model. Monthly Notices R Astronomical Soc 436(4):3366-3379. https://doi.org/10.1093/mnras/stt1818

Yeates AR, Nandy D, Mackay DH (2008) Exploring the physical basis of solar cycle predictions: flux transport dynamics and persistence of memory in advection- versus diffusion-dominated solar convection zones. Astrophysical J 673(1):544-556. https://doi.org/10.1086/524352

Yeo KL, Krivova NA, Solanki (2014) Solar cycle variation in solar irradiance. Space Sci Rev 186(1-4):137-167. https://doi.org/10.1007/s11214-014-0061-7

Yeo KL, Solanki SK, Krivova NA (2020) How faculae and network relate to sunspots, and the implications for solar and stellar brightness variations. Astronomy Astrophysics 639:A139. https://doi.org/10.1051/0004-6361/20203 7739

\section{Publisher's Note}

Springer Nature remains neutral with regard to jurisdictional claims in published maps and institutional affiliations.

\section{Submit your manuscript to a SpringerOpen ${ }^{\odot}$ journal and benefit from:}

- Convenient online submission

- Rigorous peer review

- Open access: articles freely available online

- High visibility within the field

- Retaining the copyright to your article

Submit your next manuscript at $\boldsymbol{\nabla}$ springeropen.com 